\title{
ATLAS DE LA PTERIDOFLORA IBERICA Y BALEAR
}

\author{
A. E. SALVO, B. CABEZUDO \& L. ESPAÑA \\ con la colaboración de
}

T. E. DIAZ GONZALEZ, J. IRANZO, J. IZCO \& C. PRADA

\begin{abstract}
RESUMEN: Tomando como base el Atlas Florae Europeae se ha realizado una revisión corológica de la pteridoflora ibérica y balear.

RESUME: En prennant comme base Atlas Florae Europeae nous avons réalisé una révision chorologique de la pteridoflore ibérique et balear.
\end{abstract}

\section{INTRODUCCION}

La aparición del primer volumen del Atlas Florae Europeae dedicado a los pteridófitos (Jalas \& Suominen, 1972) supuso una aportación esencial en los trabajos corológicos europeos, tanto por la novedad de la técnica empleada como por la abundancia de datos recogidos. No obstante era evidente una cierta desproporción entre los datos de densidad de especies de paises centroeuropeos y los de la Península Ibérica y Baleares (op. cit.: 11), desproporción motivada fundamentalmente por una falta de trabajos corológicos y taxonómicos sobre flora pteridofítica peninsular y balear.

Transcurridos diez años desde la aparición del primer volumen han sido numerosos los estudios pteridológicos que han completado las lagunas existentes (Salvo, Asensi \& Rivas-Martínez, 1981). Hernos creido oportuno sintetizar todos los nuevos datos y realizar una aproximación actual al conocimiento corológico de los helechos ibéricos y baleáricos, en la cual se resuma una década (1972-1982) de numerosos trabajos de pteridólogos ibéricos y extranjeros y que se refleja en la variación de los mapas de densidades del año 1972 y la del presente trabajo (Fig. 1).

Las novedades y exclusiones resultantes del presente trabajo son las siguientes: 
A) Citas nuevas

Lycopodium annotinum

Isoetes longissima

Osmunda regalis var. plumieri

Botrychium matricariifolium

Polypodium canariense

Cheilanthes guanchica

Marsilea aegyptiaca

Pteridium aquilinum subsp. brevipes

Asplenium trichomanes subsp. inexpectans

Asplenium trichomanes subsp. pachyrachis

Cystopteris viridula

Dryopteris expansa

Dryopteris remota

Dryopteris affinis subsp. affinis

Dryopteris affinis subsp. stilluppensis

Dryopteris pallida subsp. pallida

B) Nuevos táxones descritos desde 1972 y presentes en la Península Ibérica

Psilotum nudum var. molesworthae

Dryopteris guanchica

Dryopteris corleyi

Dryopteris tyrrhena

Asplenium marinum var. praealtum

Asplenium marinum var. depauperatum

C) Táxones a excluir de la flora ibérica

Isoetes brochonii Asplenium obovatum

Isoetes boryana

Asplenium cuneifolium

Un trabajo de esta índole nunca es definitivo, por lo cual esperamos que las seguras omisiones sean subsanadas por las aportaciones de futuros trabajos. Para evitar la dispersión bibliográfica de los mismos la dirección de ACTA BOTANICA MALACITANA se ofrece a publicar períodicamente todas las novedades corológicas y taxonómicas de helechos peninsulares y baleáricos con el nombre de Notas Pteridológicas de la Península Ibérica y Baleares.
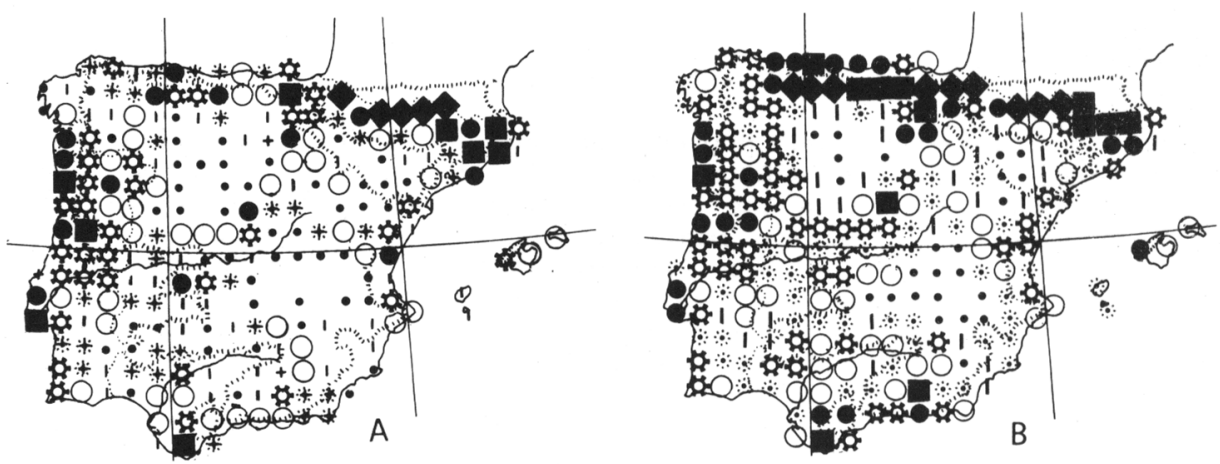

Figura 1.- Comparación de la densidad de táxones de pteridófitos en cuadriculas de $50 \mathrm{~km}$ de lado en la Peninsula Ibérica y Baleares, entre el mapa presentado por Jalas y Suominen en 1972 (A) y el obtenido en el presente trabajo (B). Clave de densidades: (•):1-3; (I):4-7; (*):

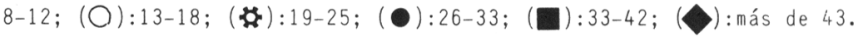




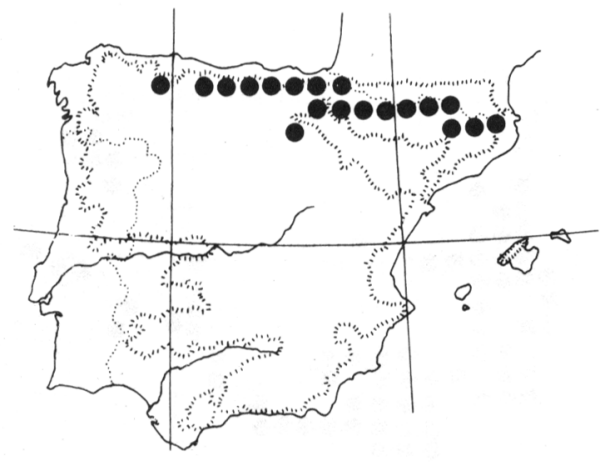

1. Huperzia selago L.

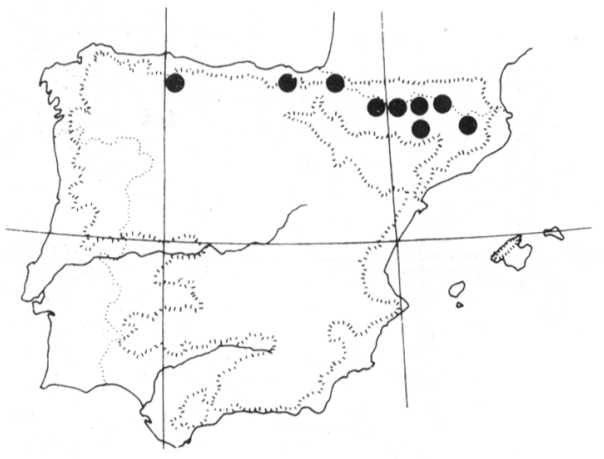

3. Diphasiastrum alpinum (L.) Rothm.

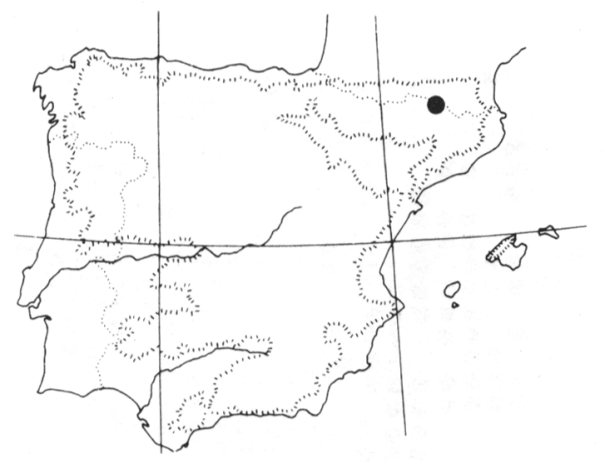

5. Lycopodium annotinum L.

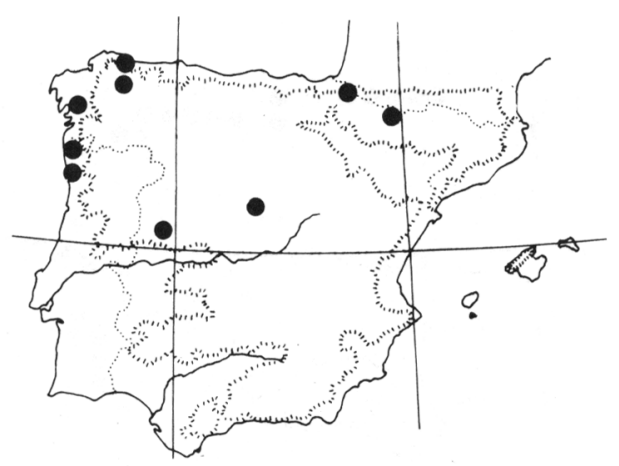

2. Lepidotis inundata (L.) Börner

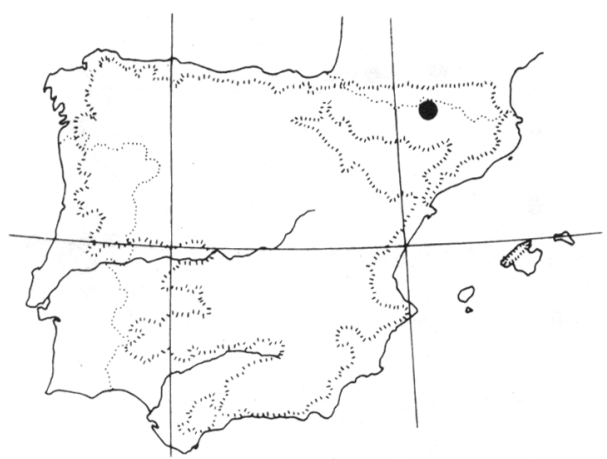

4. Diphasiastrum complanatum (L.) Holub

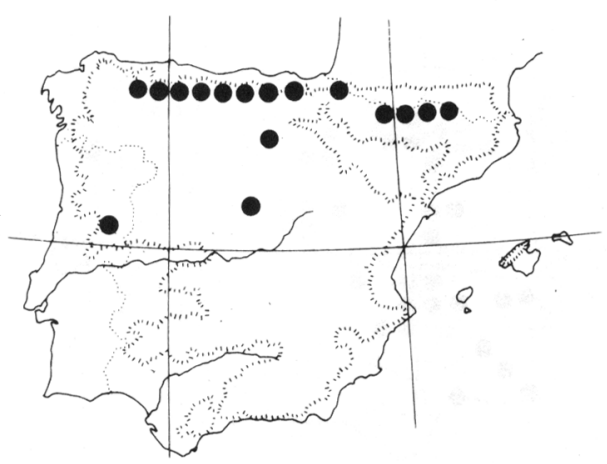

6. Lycopodium clavatum L. 


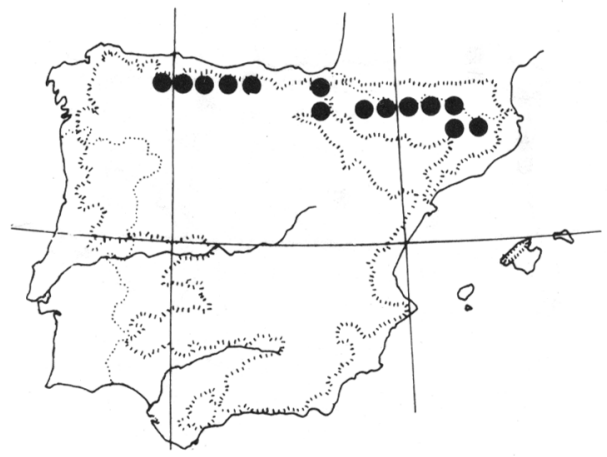

7. Selaginella selaginoides (L.) Link

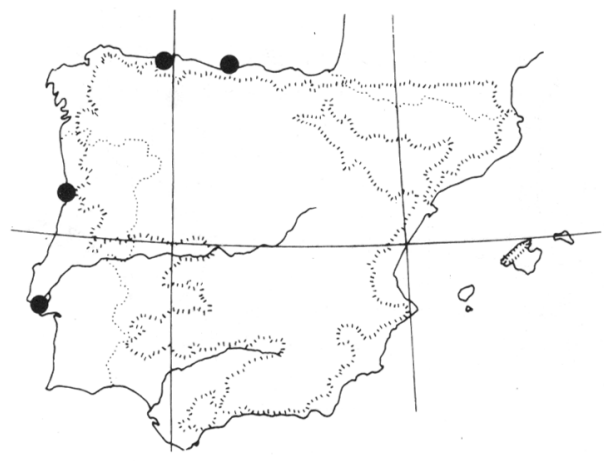

9. Selaginella kraussiana (G. Kunze) A. Braun

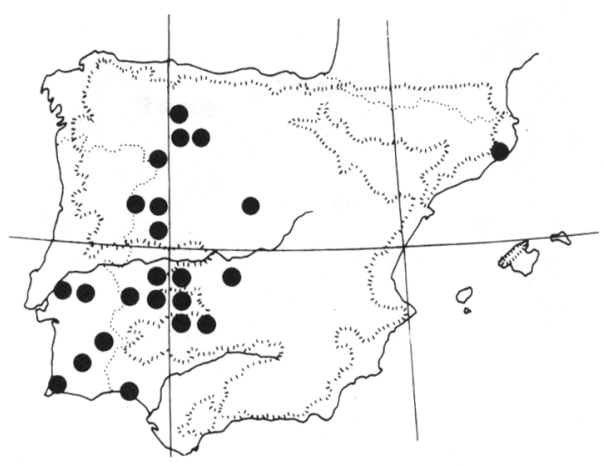

11. Isoetes setacea Lam.

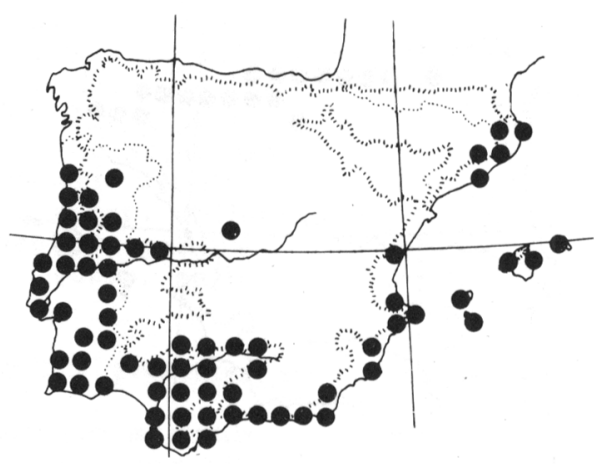

8. Selaginella denticulata (L.) Link

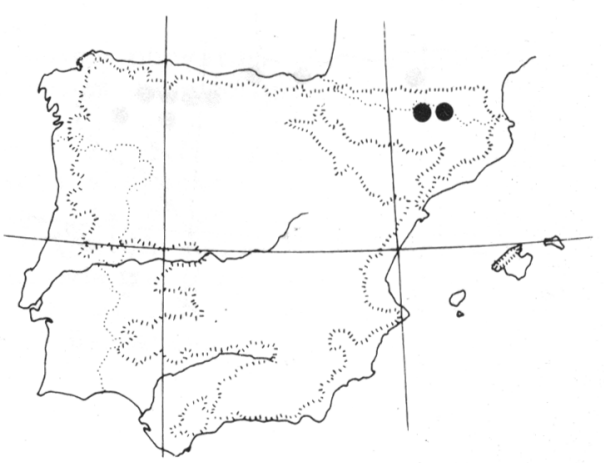

10. Isoetes lacustris L.

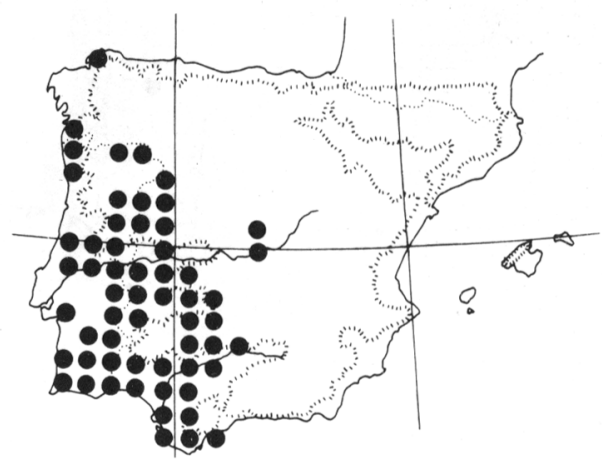

12. Isoetes histrix Bory 


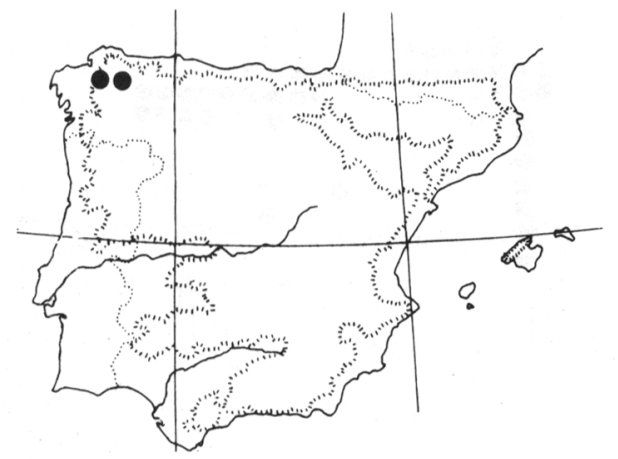

13. Isoetes longissima Bory

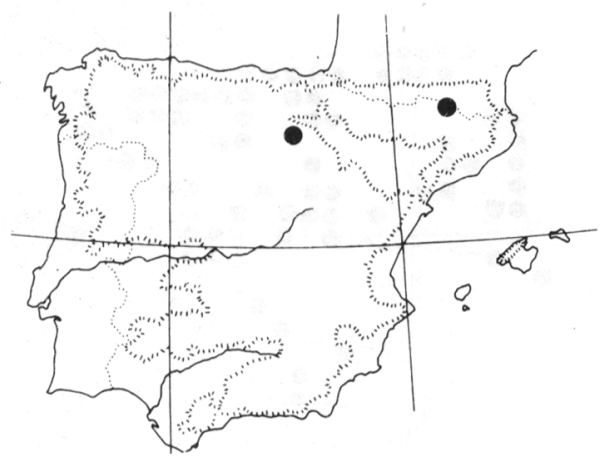

15. Isoetes echinospora Durieu

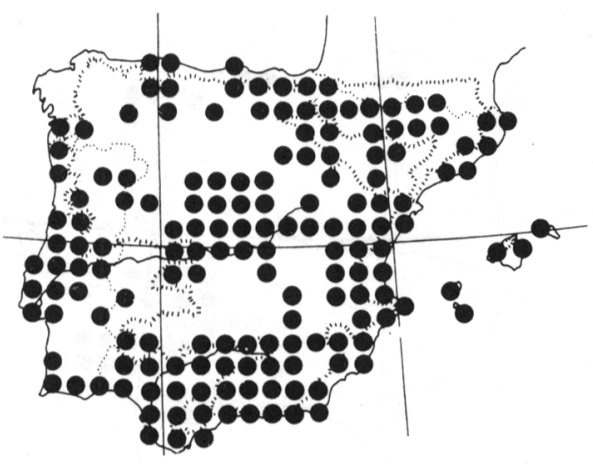

17. Equisetum ramosissimum Desf.

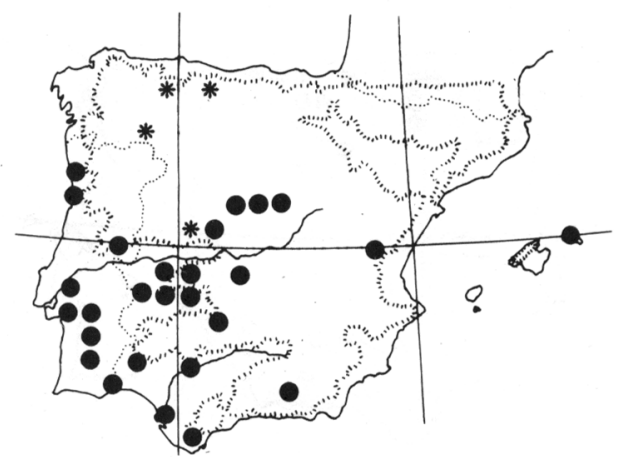

14. Isoetes velata A. Braun subsp. velata ( ) ; subsp. asturicense (Lainz) Riv.-Mart. \& Prada (*)

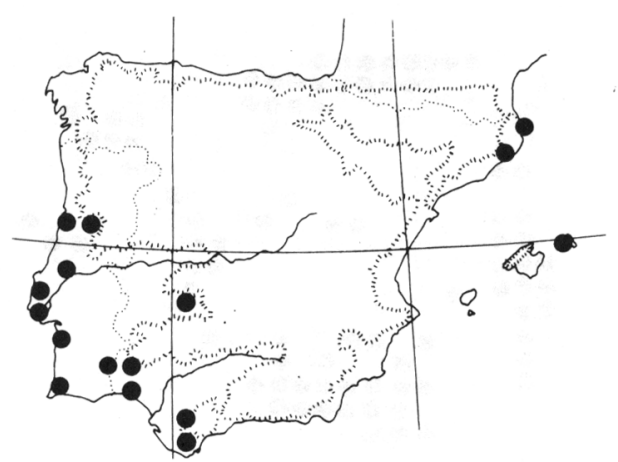

16. Isoetes durieui Bory

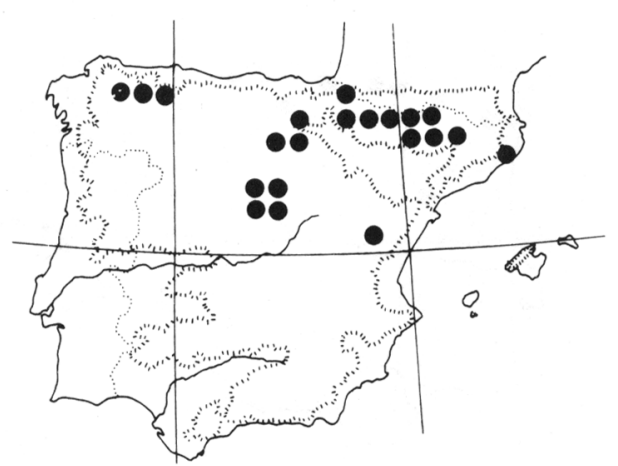

18. Equisetum hyemale L. 


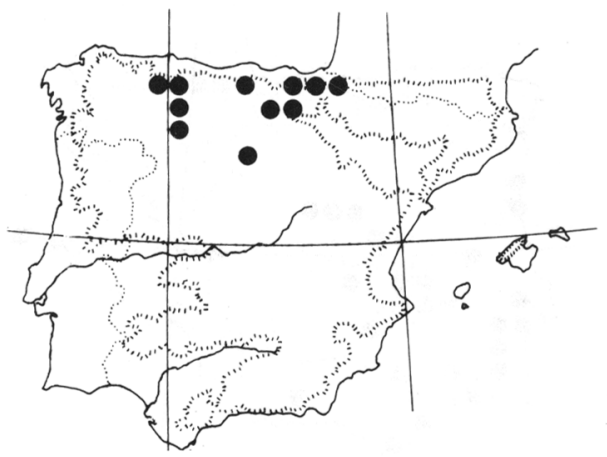

19. Equisetum fluviatile L.

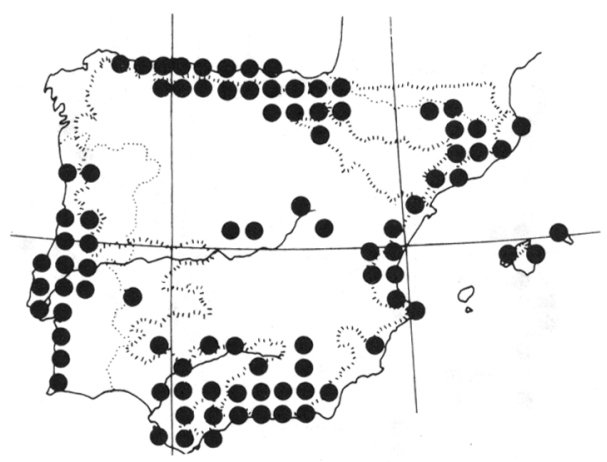

21. Equisetum telmateia Ehrh.

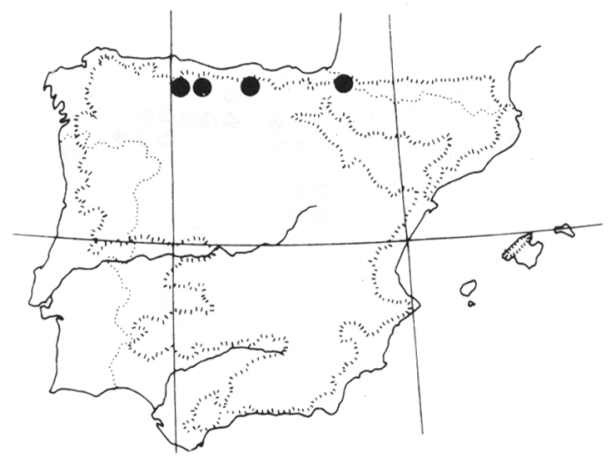

23. Equisetum sylvaticum L.

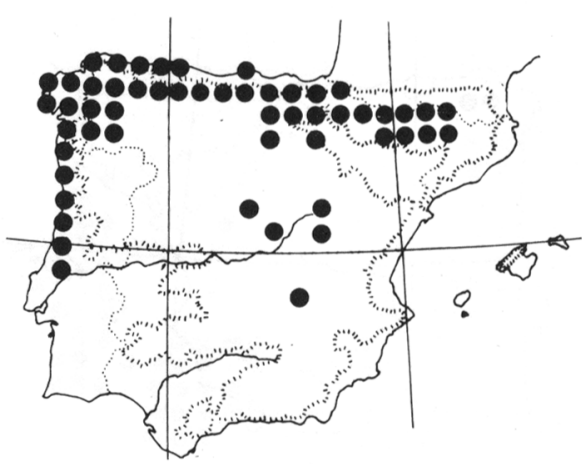

20. Equisetum palustre L.

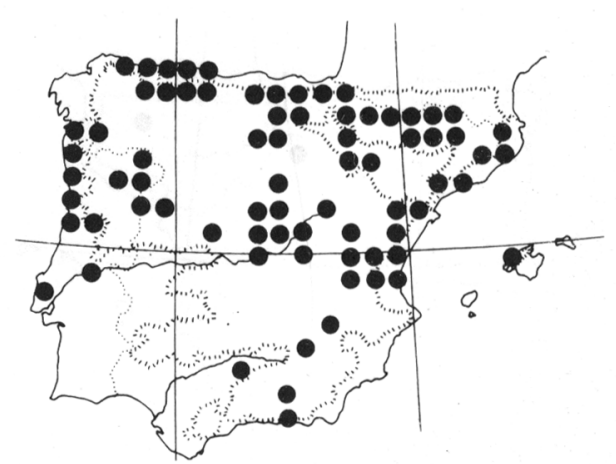

22. Equisetum arvense L.

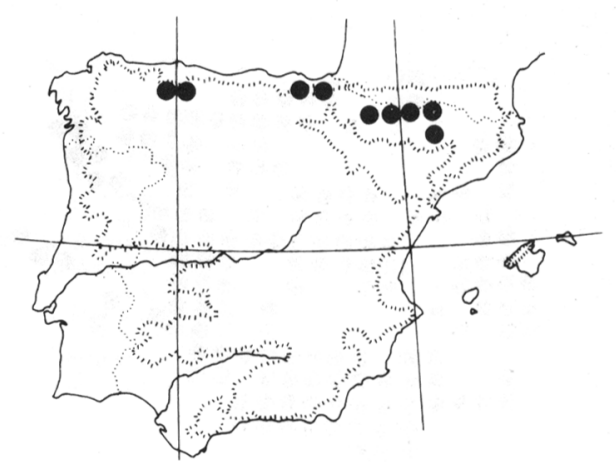

24. Equisetum variegatum Schleicher 


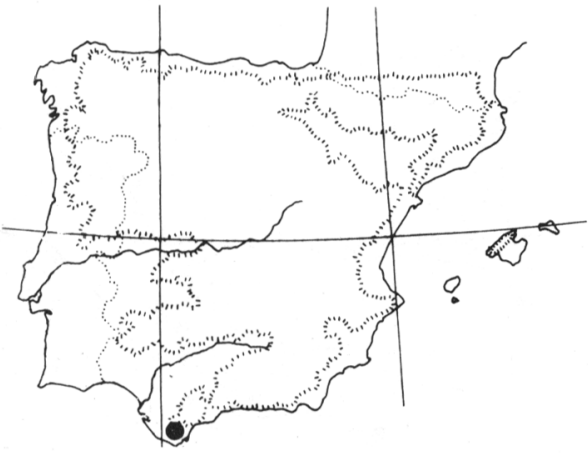

25. Psilotum nudum (L.) P. Beauv. var. molesworthae Iranzo, Prada \& Salvo

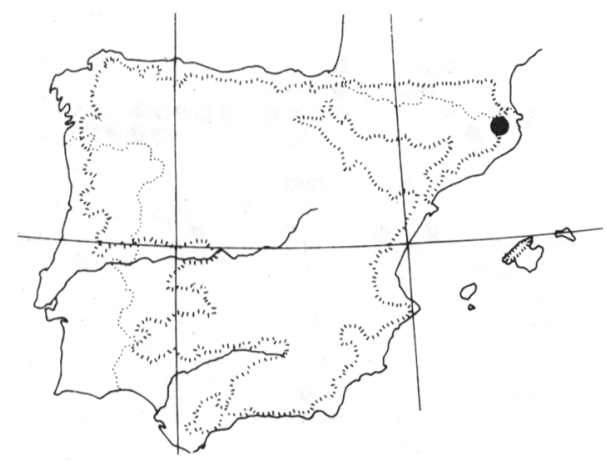

27. Botrychium matricarifolium Koch

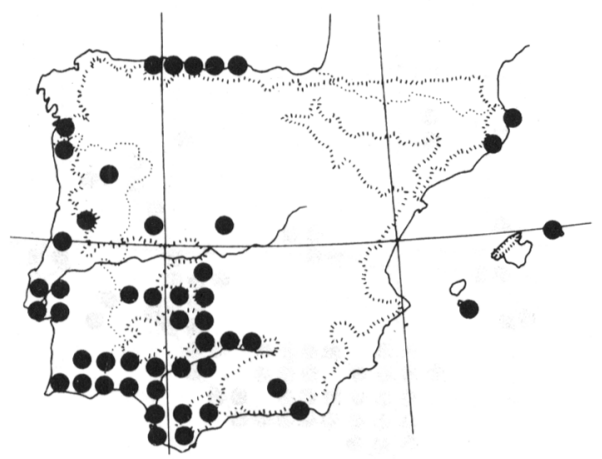

29. Ophioglossum lusitanicum L.

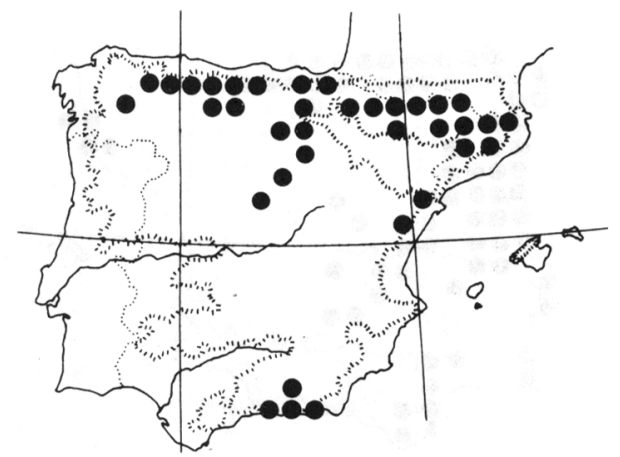

26. Botrychium lunaria (L.) Swartz

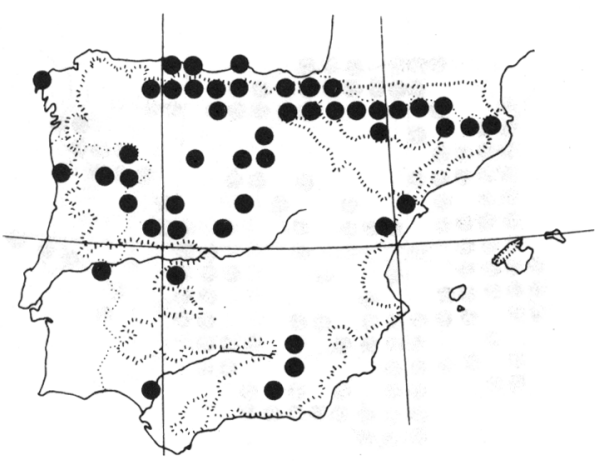

28. Ophioglossum vulgatum L.

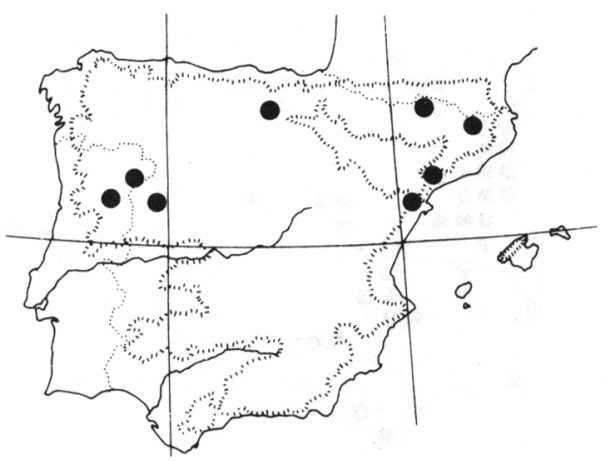

30. Ophioglossum azoricum C. Presl. 


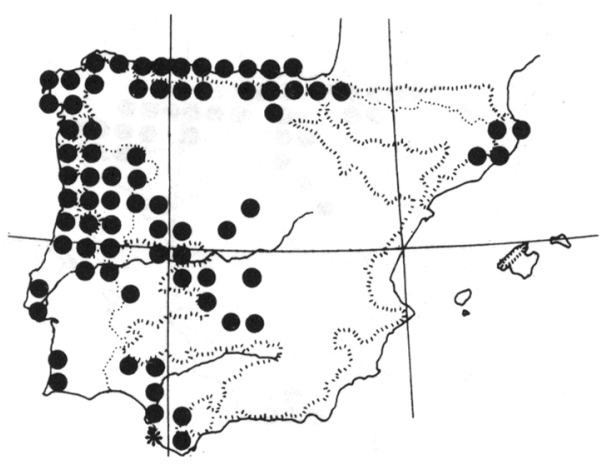

31. Osmunda regalis L.

var. regalis (•); var. plumieri ( Tausch) Milde (*).

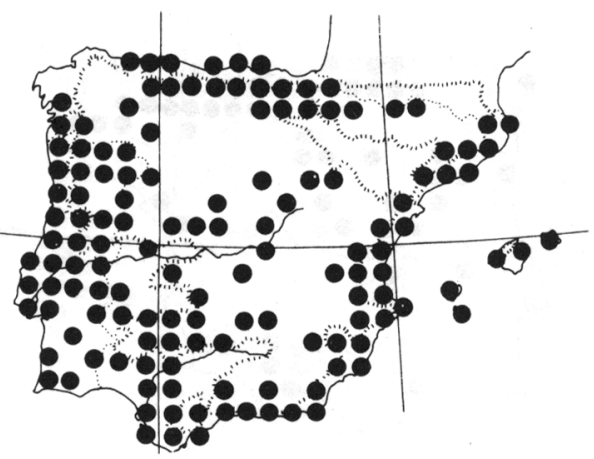

33. Polypodium cambricum L.

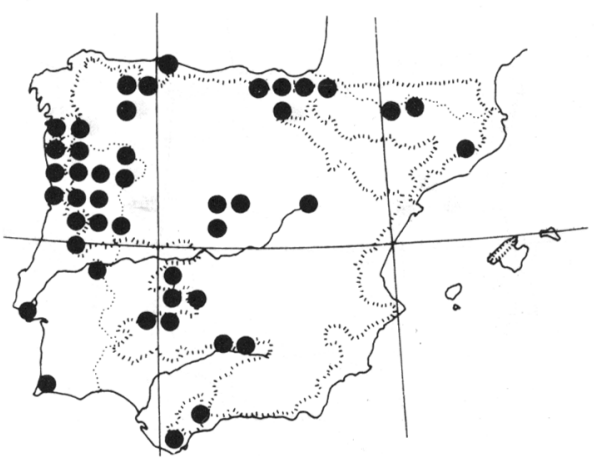

35. Polypodium interjectum Shivas

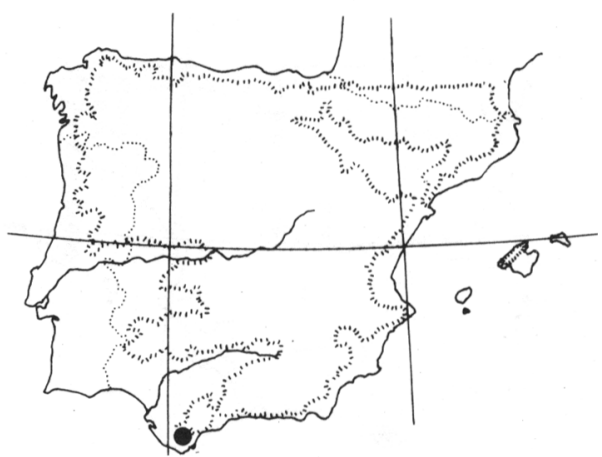

32. Polypodium canariense Willd.

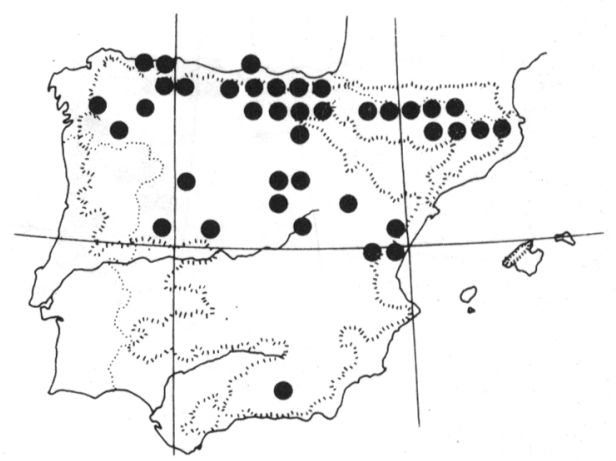

34. Polypodium vulgare L.

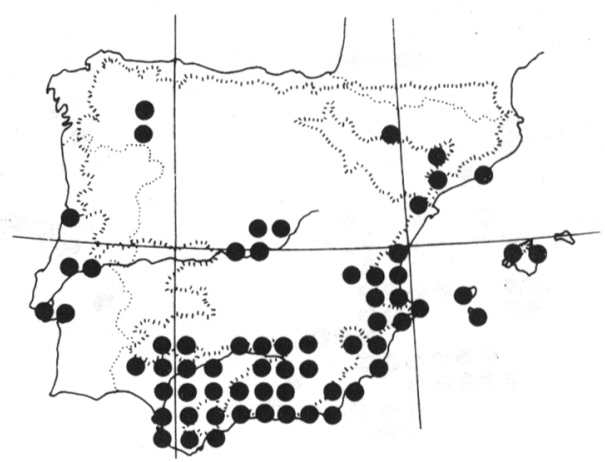

36. Cheilanthes pteridioides (Reichard) C. Chr. 


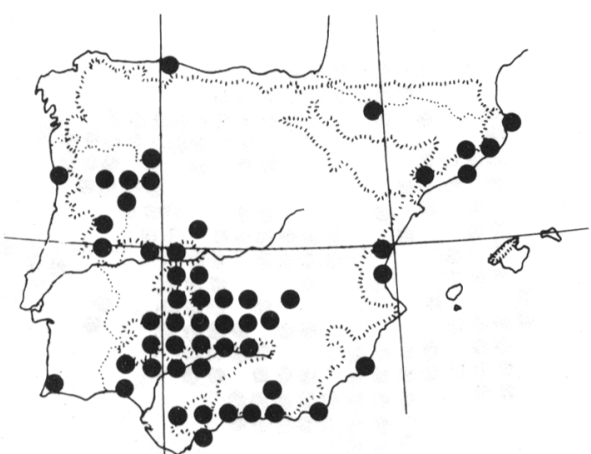

37. Cheilanthes maderensis Lowe

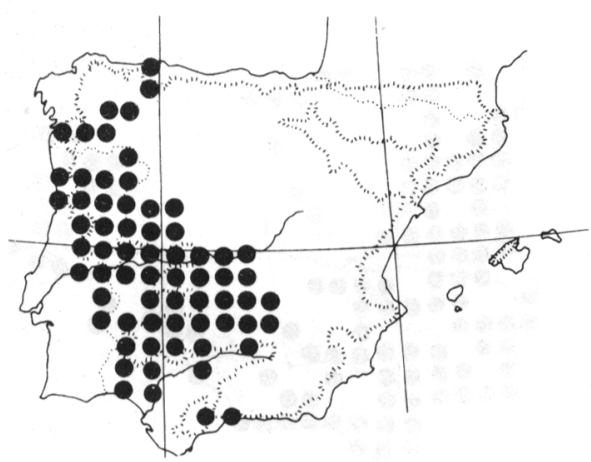

39. Cheilanthes hispanica Mett.

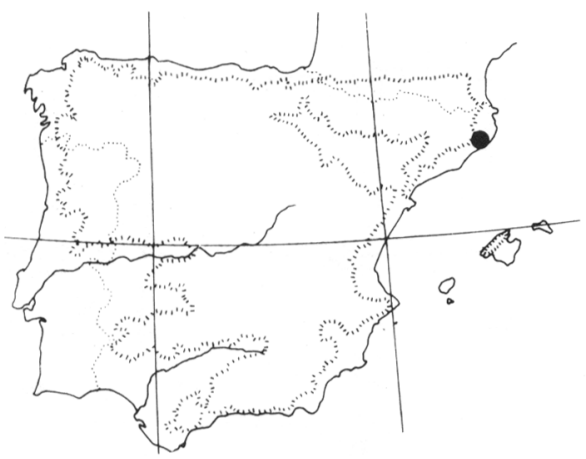

11. Pellaea calomelamos (Swartz) Link

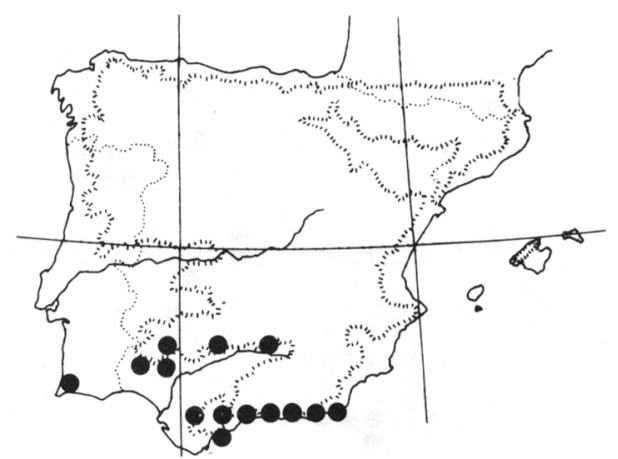

38. Cheilanthes guanchica Bolle

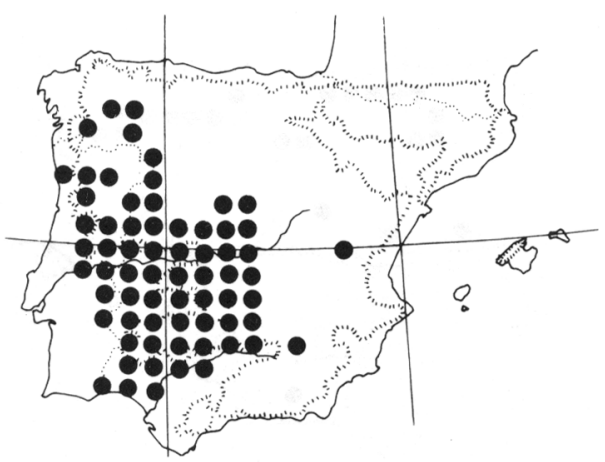

40. Cheilanthes tinaei Tod.

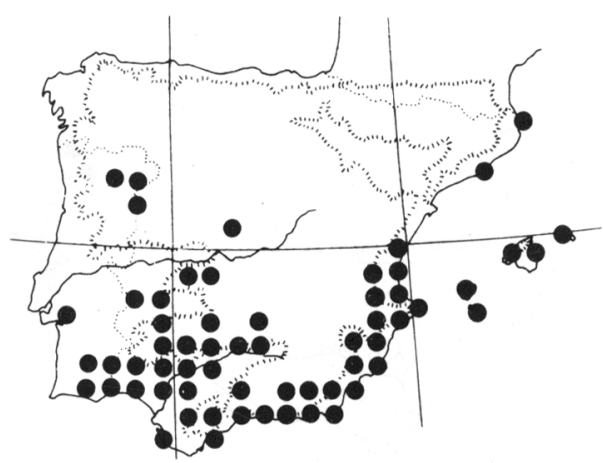

42. Notholaena vellea (Aiton) R. Br. 


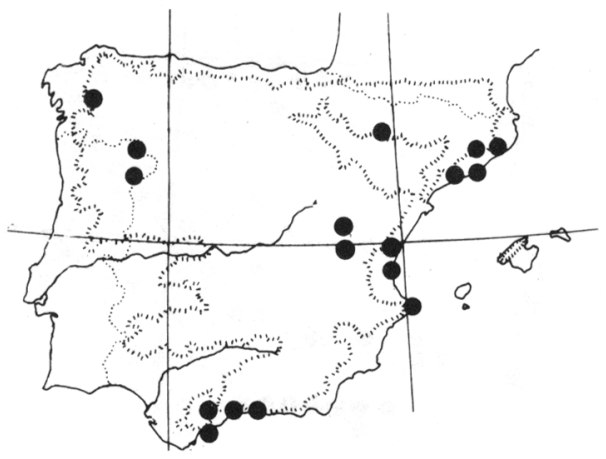

43. Notholaena marantae (L.) Desv.

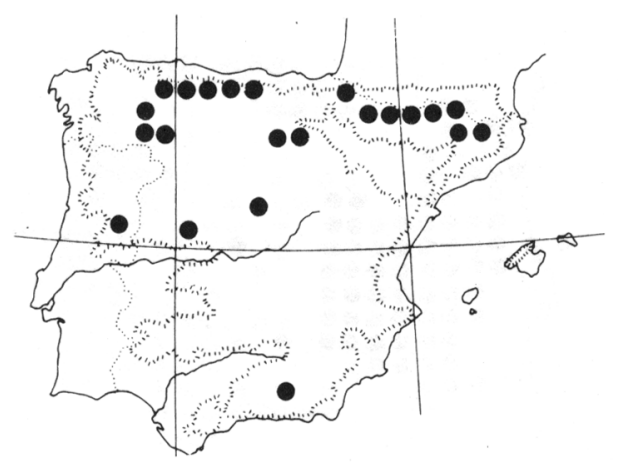

45. Cryptogramma crispa (L.) Hooker

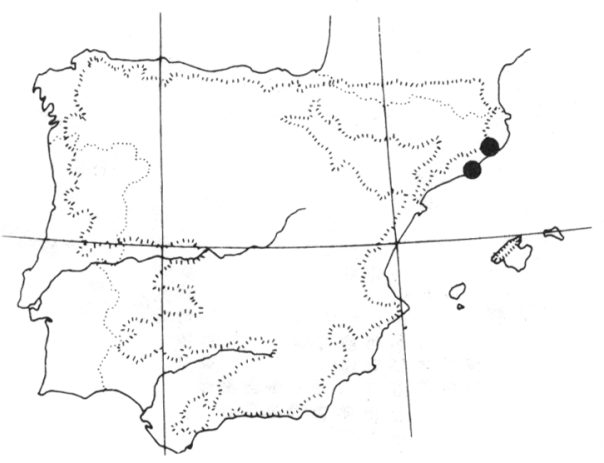

47. Pteris cretica L.

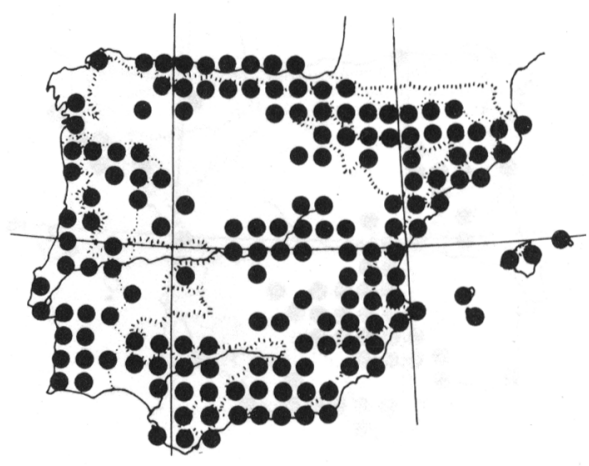

44. Adiantum capillus-veneris L.

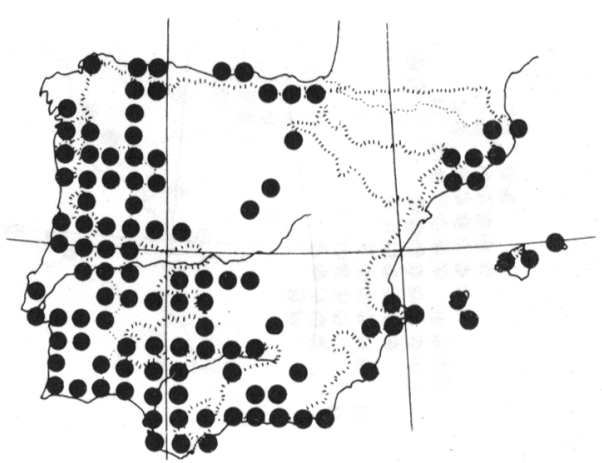

46. Anogramma leptophylla (L.) Link

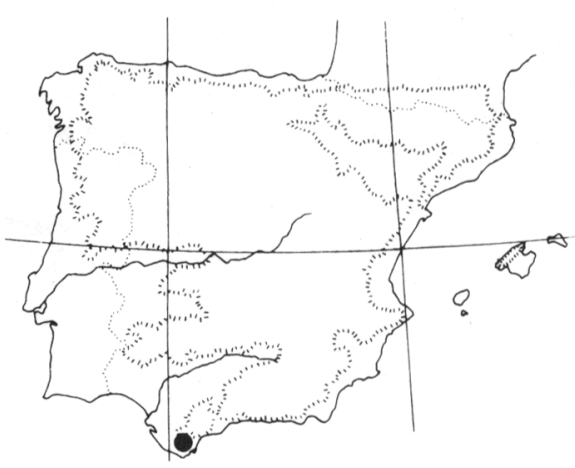

48. Pteris palustris Poiret 


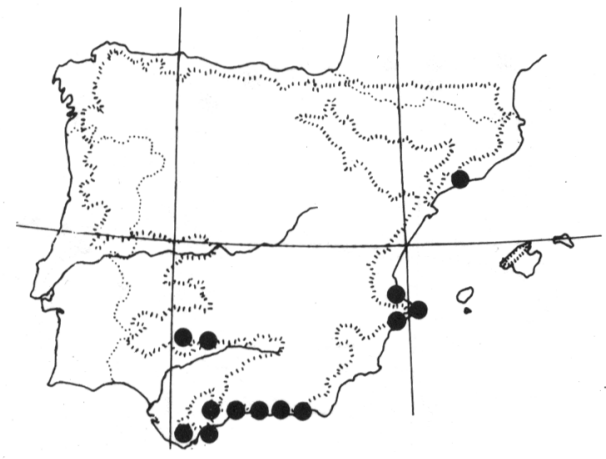

49. Pteris vittata L.

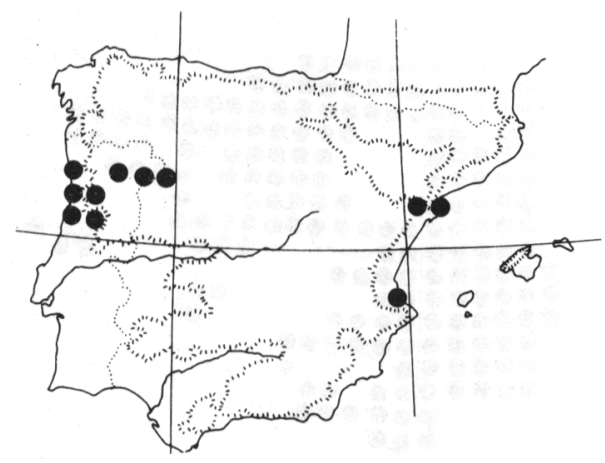

51. Marsilea quadrifolia L.

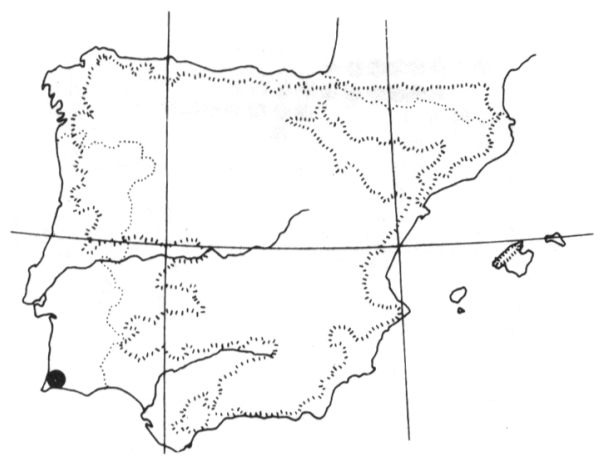

53. Pilularia minuta L.

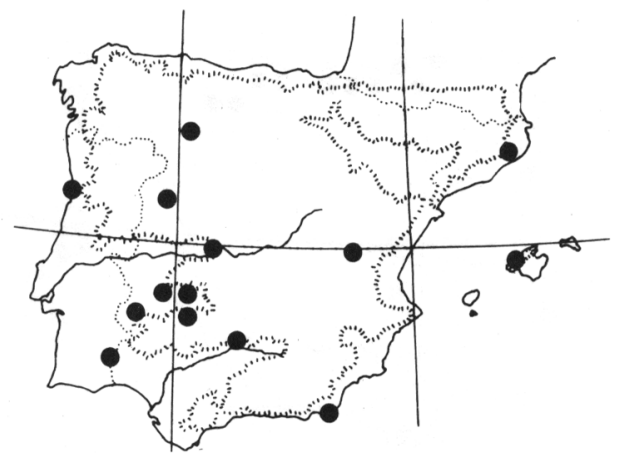

50. Marsilea strigosa Willd.

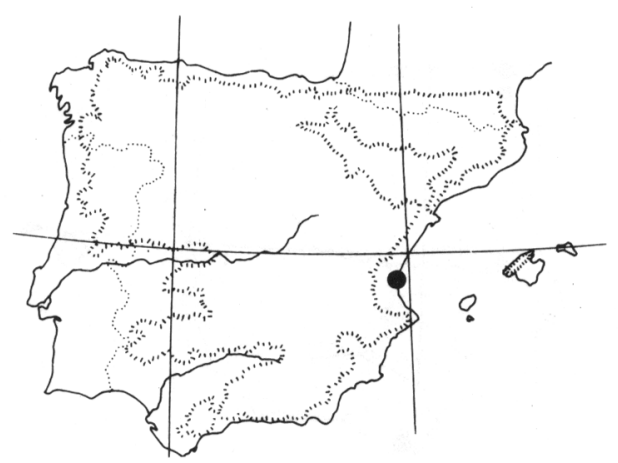

52. Marsilea aegyptiaca Willd.

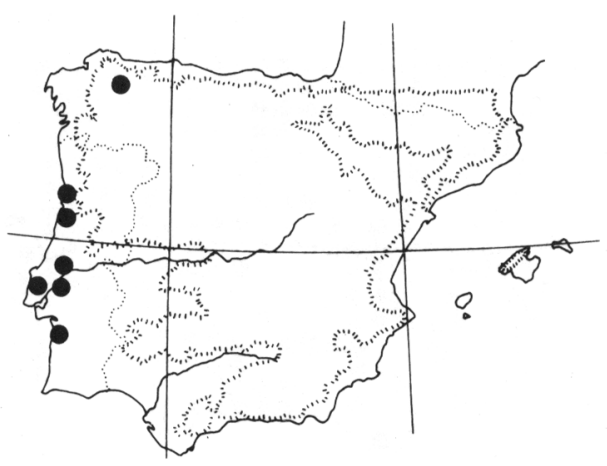

54. Pilularia globulifera L. 


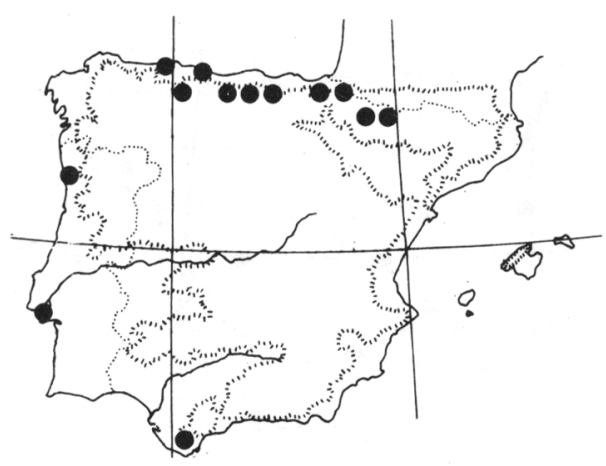

55. Vandenboschia speciosa (Willd.) Kunkel

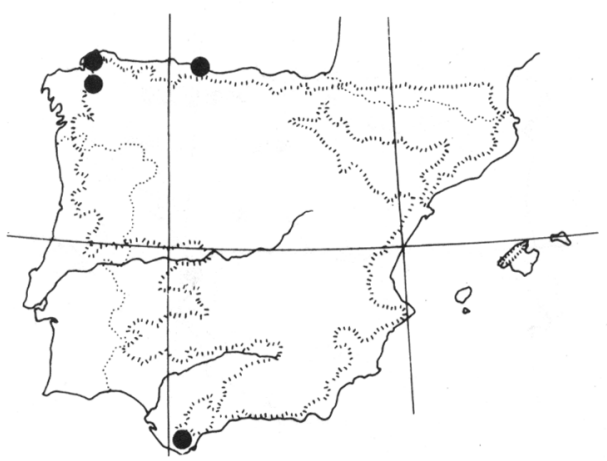

57. Culcita macrocarpa C. Presl.

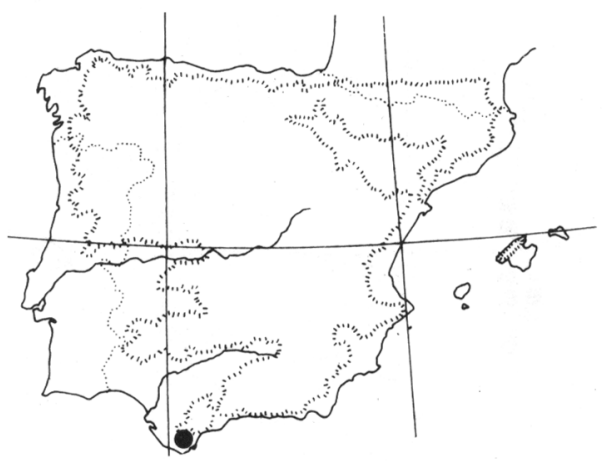

59. Christella dentata (Forsskal) Brownsey \& Jermy

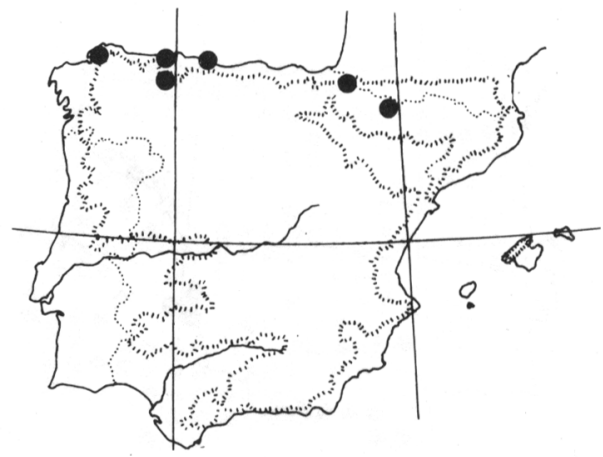

56. Hymenophyllum tunbrigense (L.) Sm.

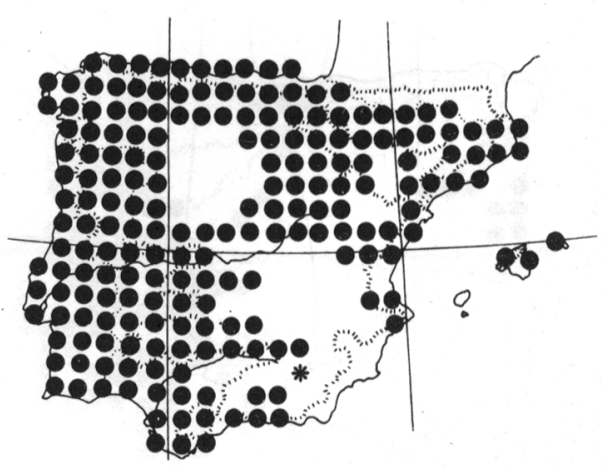

58. Pteridium aquilinum (L.) Kuhn subsp. aquilinum ( ); subsp. brevipes (Tausch) Vul'f (*).

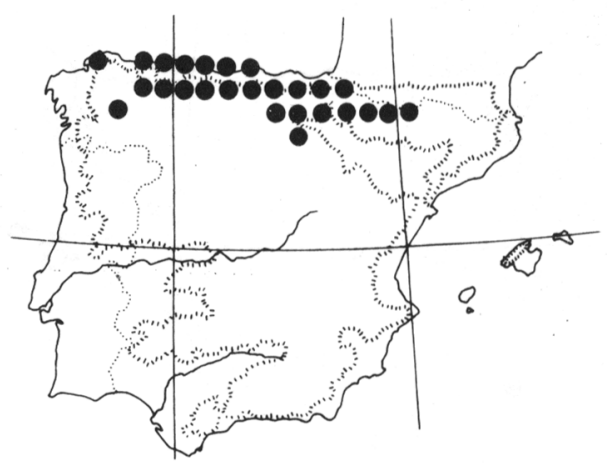

60. Oreopteris limbosperma (All.) Holub 


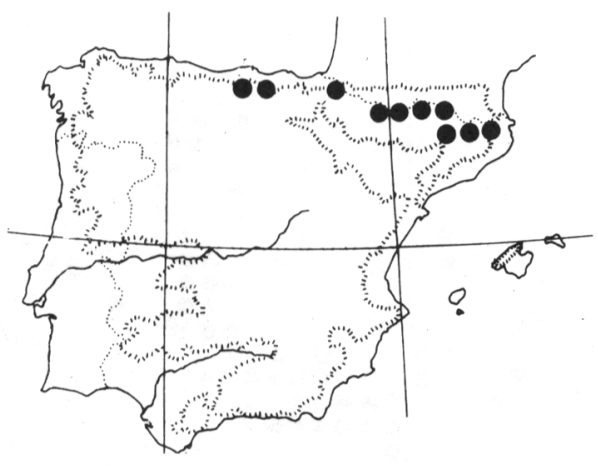

61. Phegopteris connectilis (Michx.) Watt.

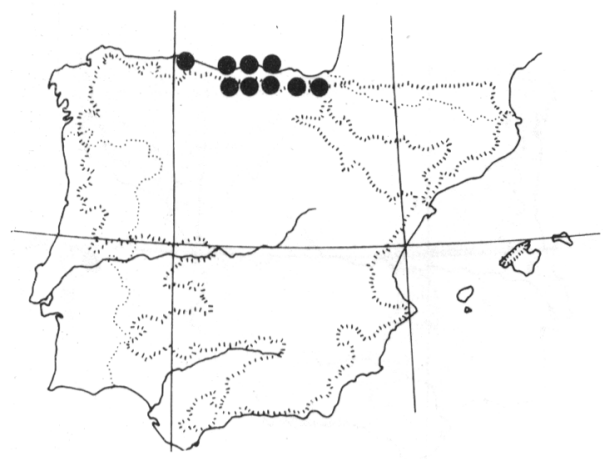

63. Stegnogramma pozoi (Lag.) Iwatsuki

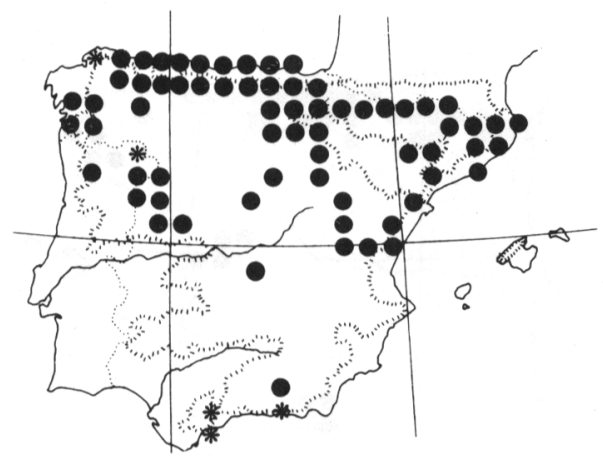

65. Asplenium adiantum-nigrum L. var. adiantum-nigrum (•); var. corunnense Merino (*).

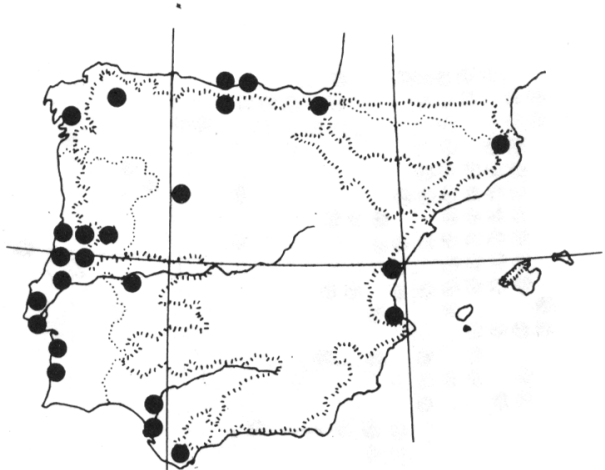

62. Thelypteris palustris Schott

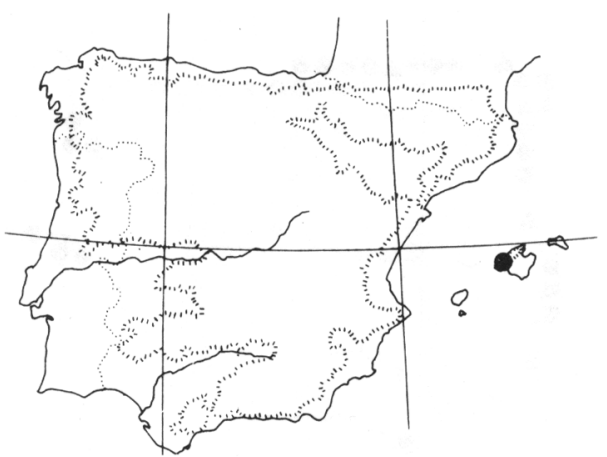

64. Asplenium balearicum Shivas

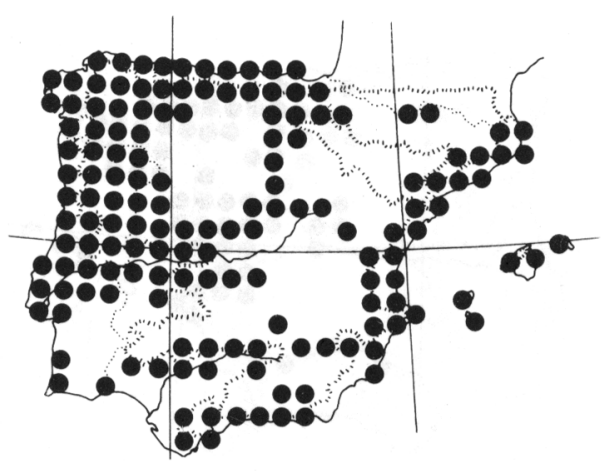

66. Asplenium onopteris L. 


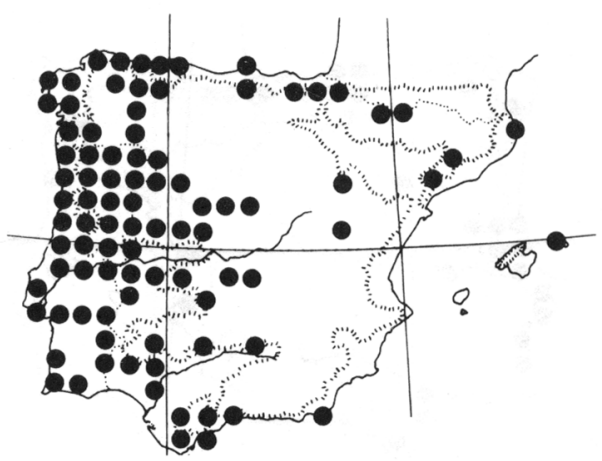

67. Asplenium billotii Schultz

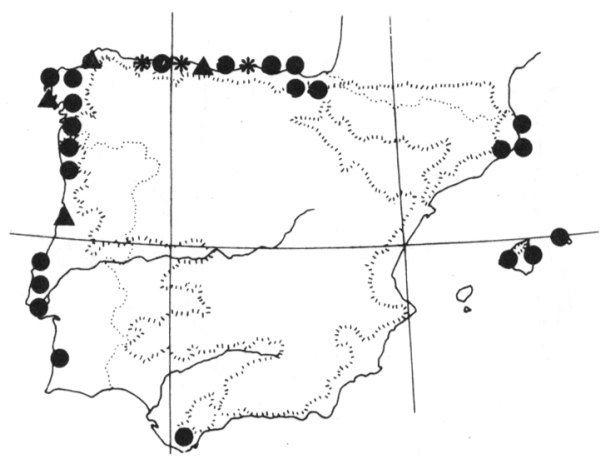

69. Asplenium marinum L.

var. marinum (-); var. praealtum

Diaz \& Salvo (*); var. depauperatum Diaz \& Salvo $(\boldsymbol{\Delta})$.

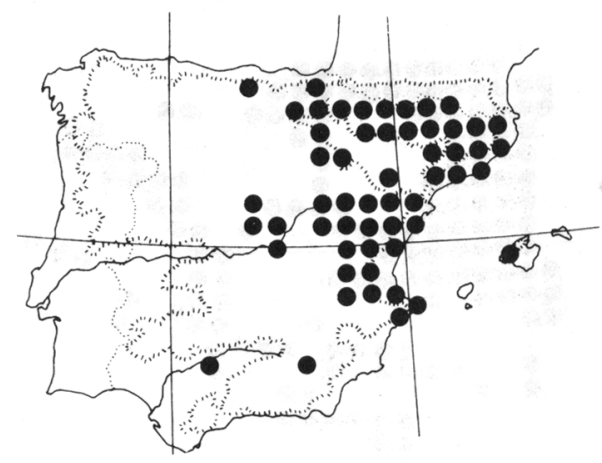

71. Asplenium fontanum (L.) Bernh.

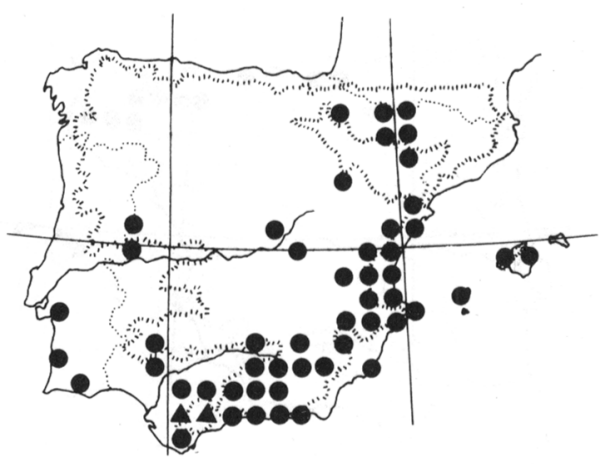

68. Asplenium petrarchae (Guérin) DC. subsp. petrarchae (๑); subsp. bivalens (Meyer) Lovis \& Reichstein ( $\mathbf{A}$ ).

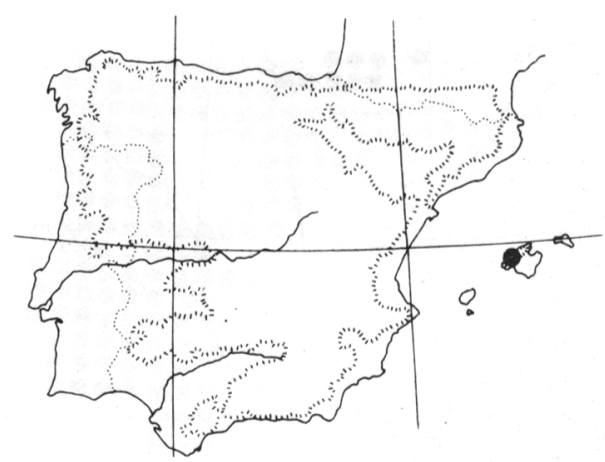

70. Asplenium majoricum Litard.

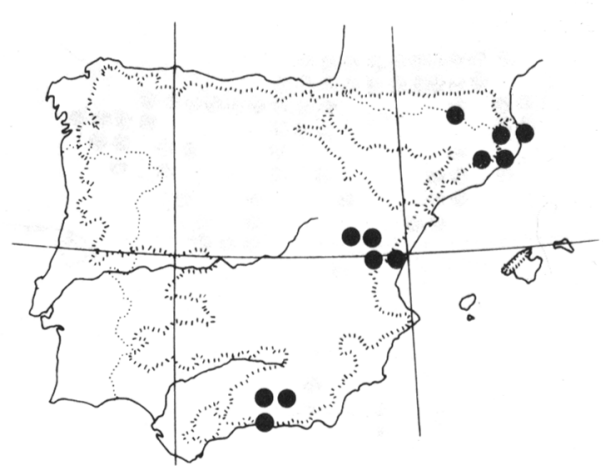

72. Asplenium foreziense Héribaud 


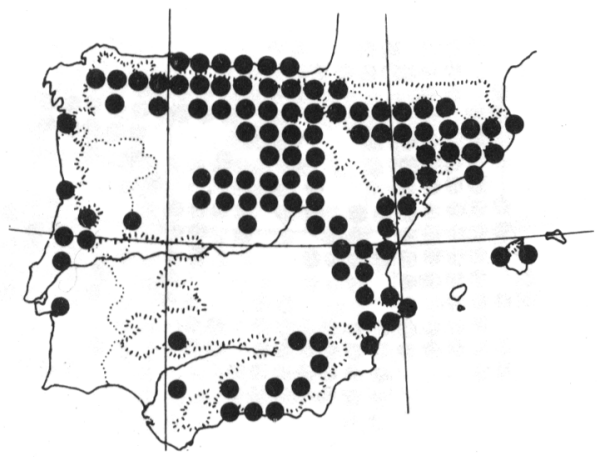

73. Asplenium ruta-muraria L.

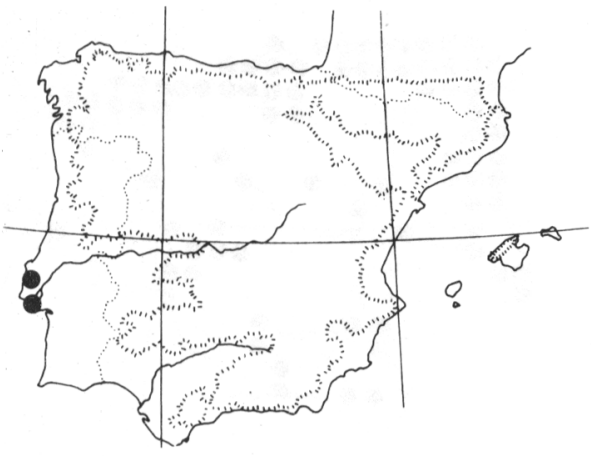

75. Asplenium hemionitis L.

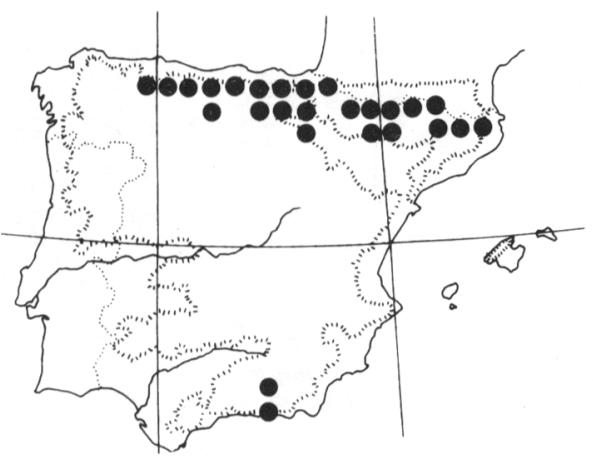

77. Asplenium viride Hudson

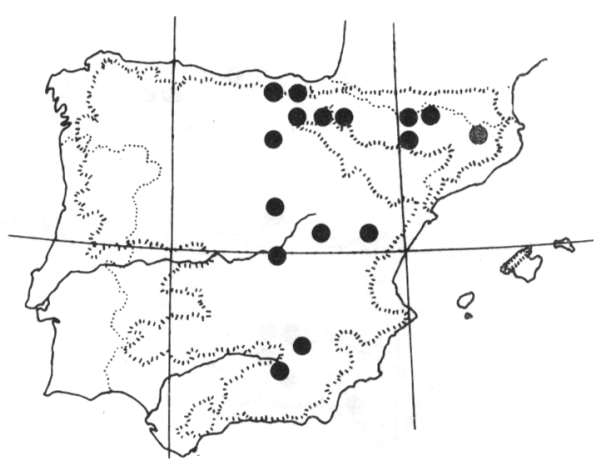

74. Asplenium celtibericum Riv.-Mart.

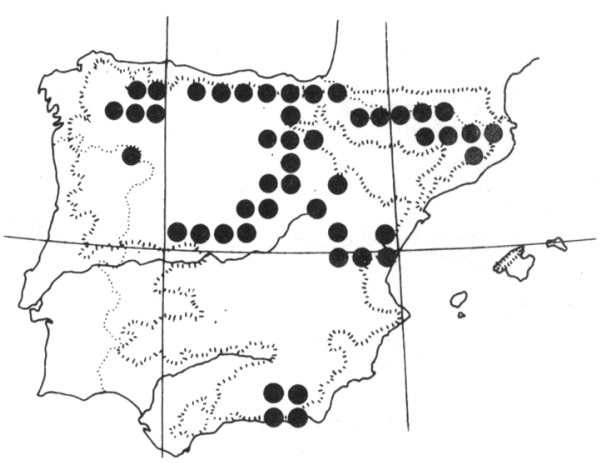

76. Asplenium septentrionale (L. ) Hoffm.

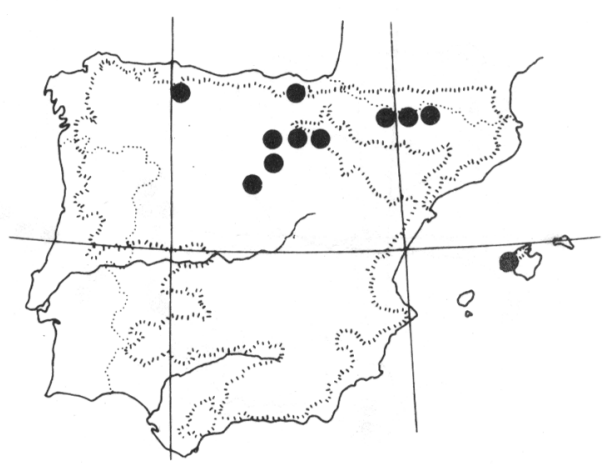

78. Asplenium trichomanes L. subsp. trichomanes 


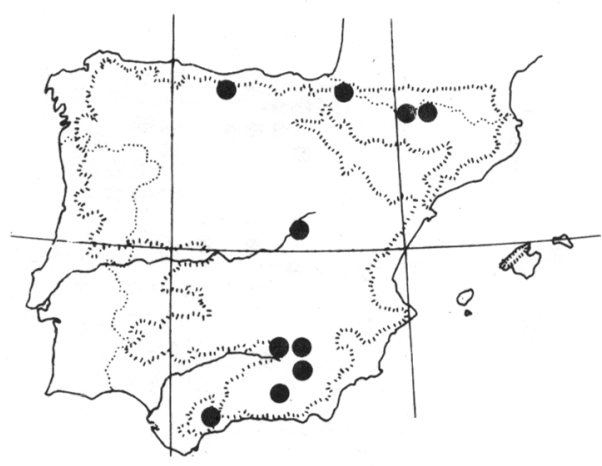

79. Asplenium trichomanes L. subsp. pachyrachis (Christ.) Lovis \& Reichstein

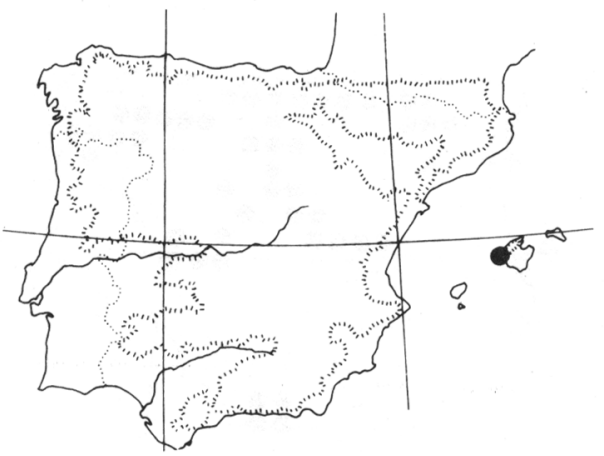

81. Asplenium trichomanes L. subsp. inexpectans Lovis

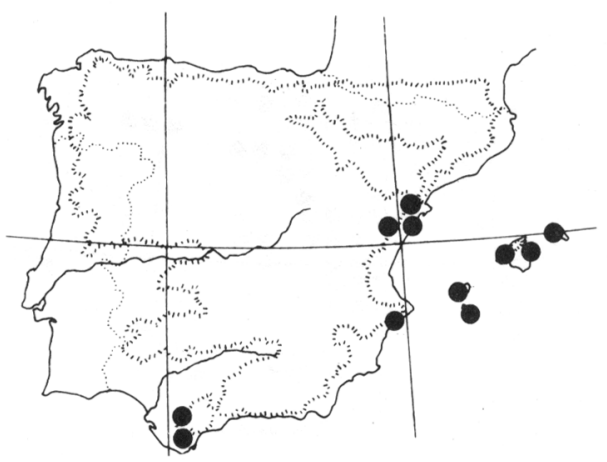

83. Asplenium sagittatum (DC.) Bange

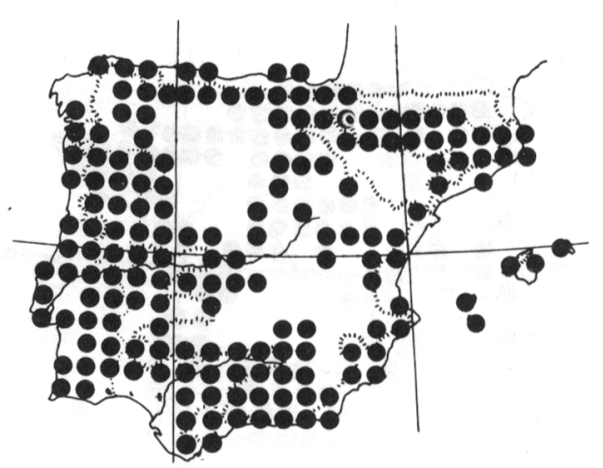

80. Asplenium trichomanes L. subsp. quadrivalens Meyer

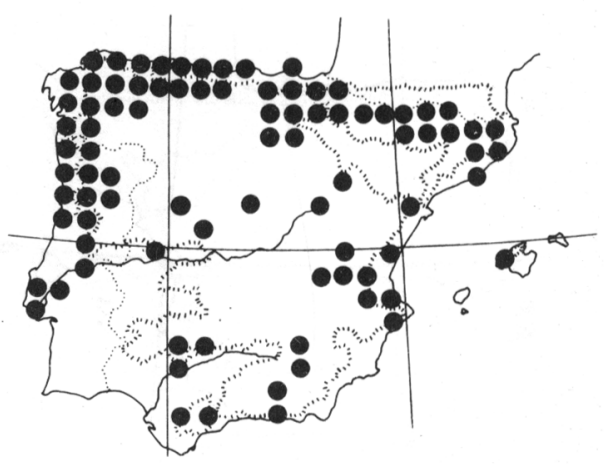

82. Asplenium scolopendrium L.

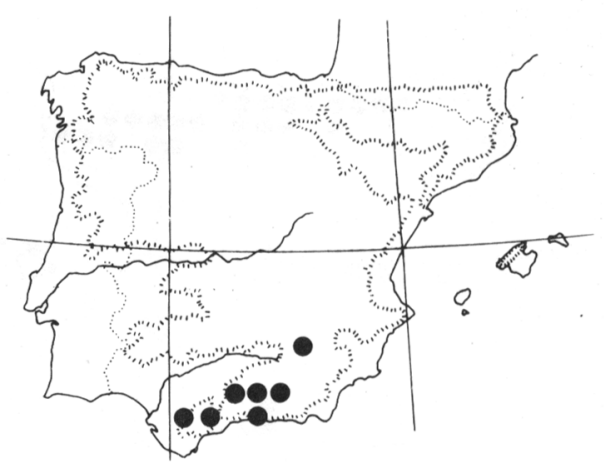

84. Asplenium subglandulosum (Hooker \& Grev.) Salvo, Prada \& Diaz subsp. hispanicum (Cosson) Salvo, Prada \& Diaz 


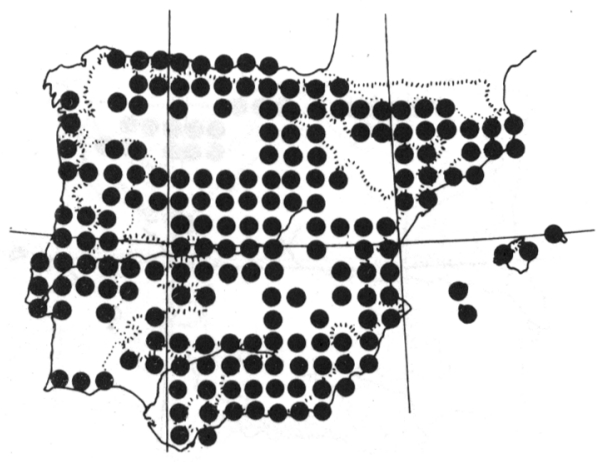

85. Asplenium ceterach L.

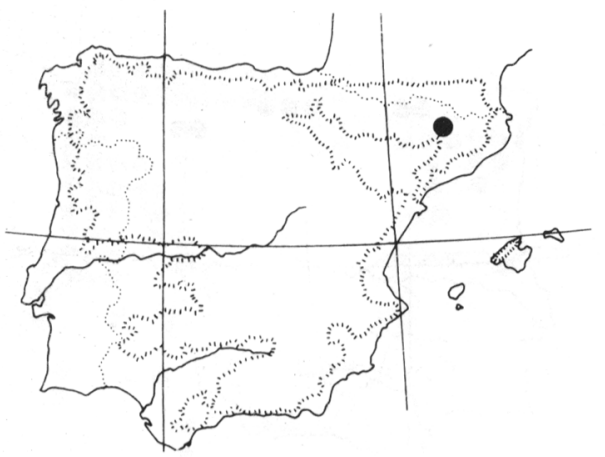

87. Woodsia pulchella Bertol.

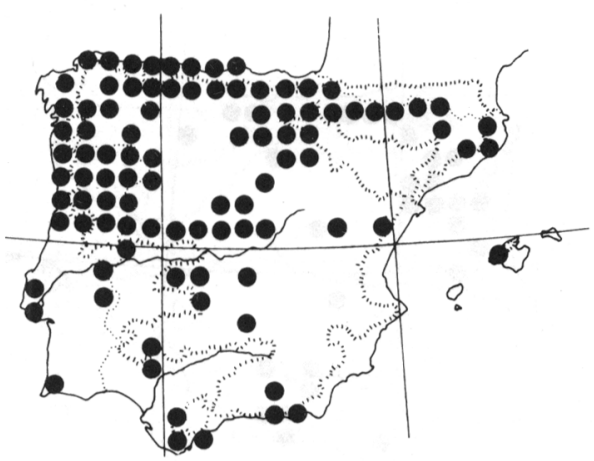

89. Athyrium filix-femina (L.) Roth

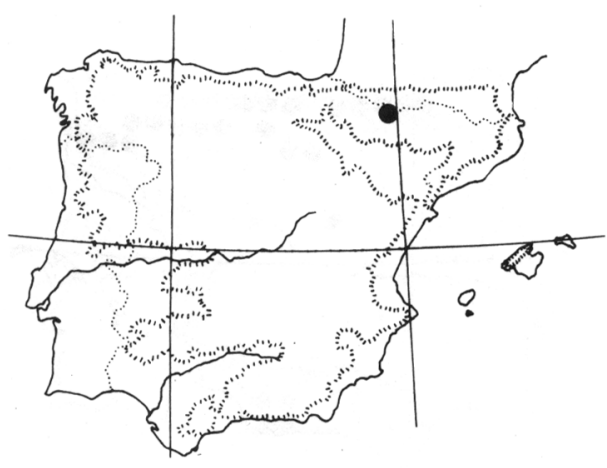

86. Woodsia alpina (Bolton) S.F. Gray

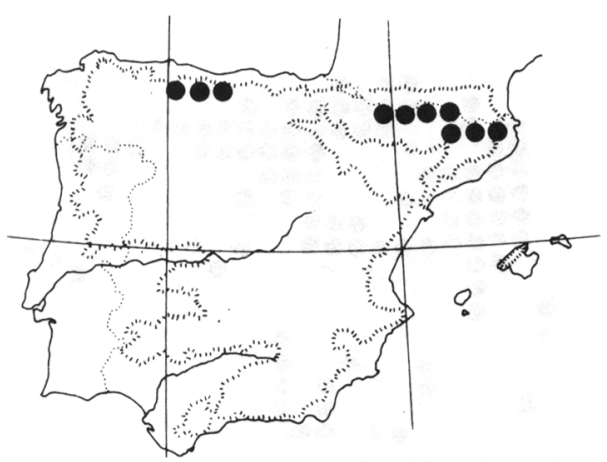

88. Athyrium distentifolium Opiz

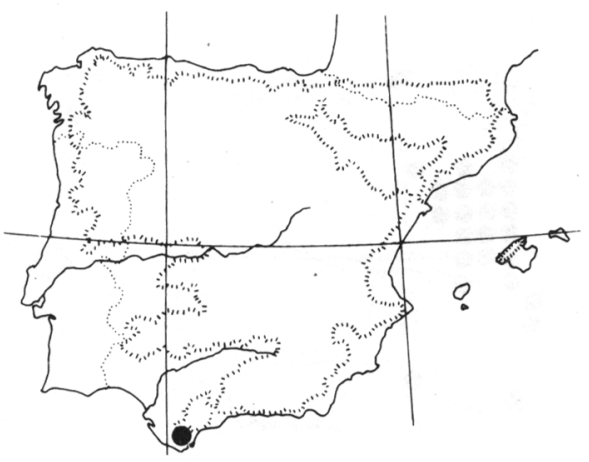

90. Diplazium caudatum (Cav.) Jermy 


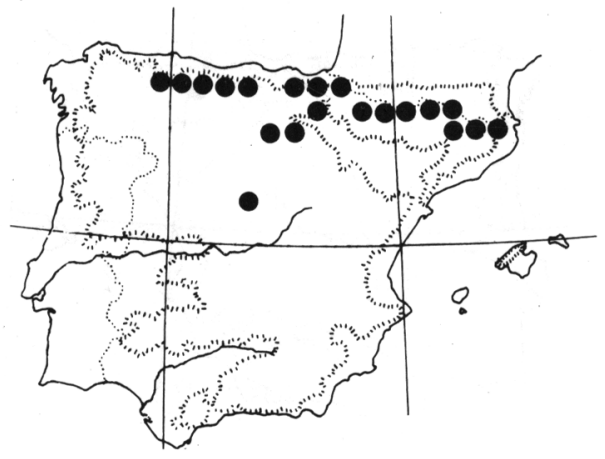

91. Gymnocarpium dryopteris (L.) Newman

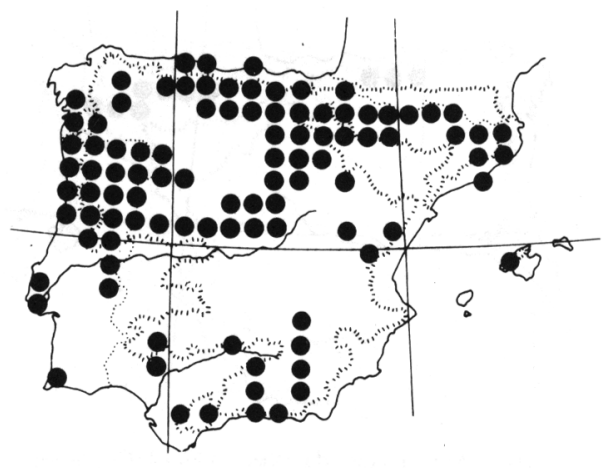

93. Cystopteris fragilis (L.) Bernh. subsp. fragilis

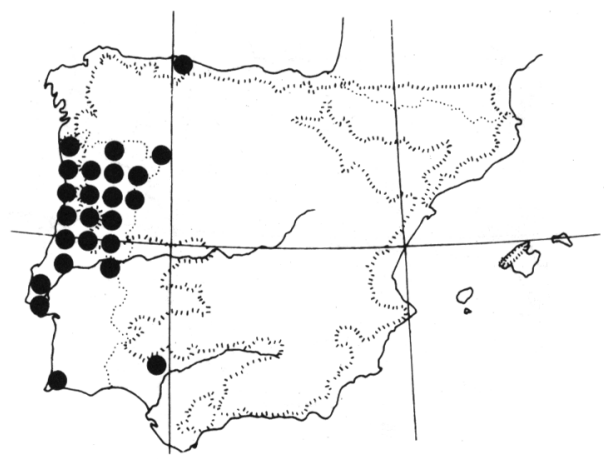

95. Cystopteris viridula (Desv.) Desv.

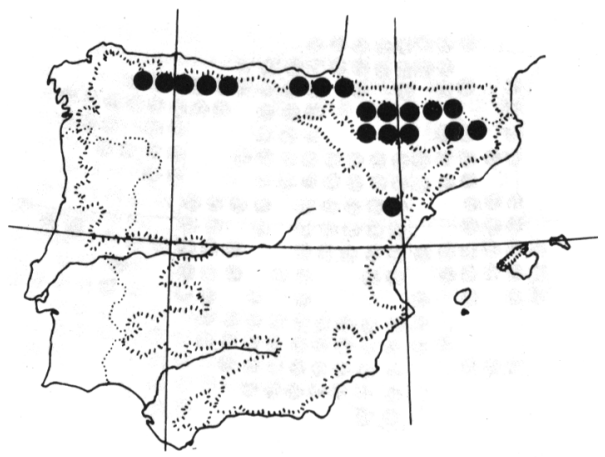

92. Gymnocarpium robertianum (Hoffm.) Newman

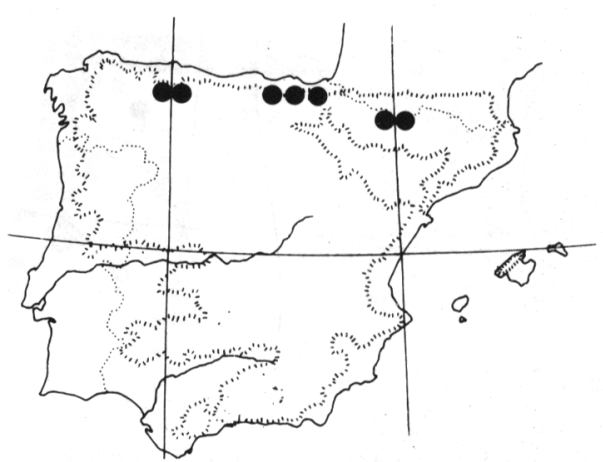

94. Cystopteris fragilis (L.) Bernh. subsp. alpina (Lam.) Briquet

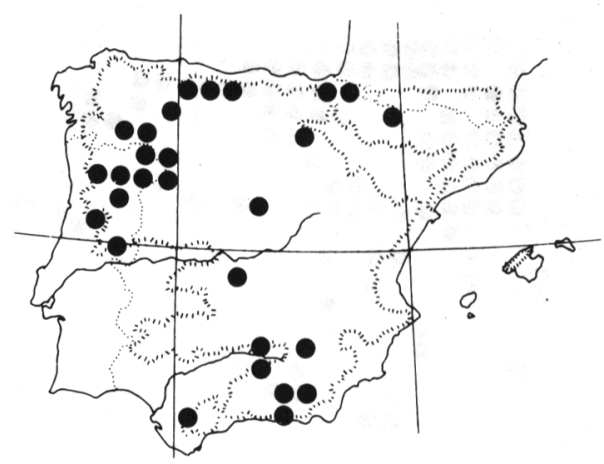

96. Cystopteris dickieana R. Sim. 


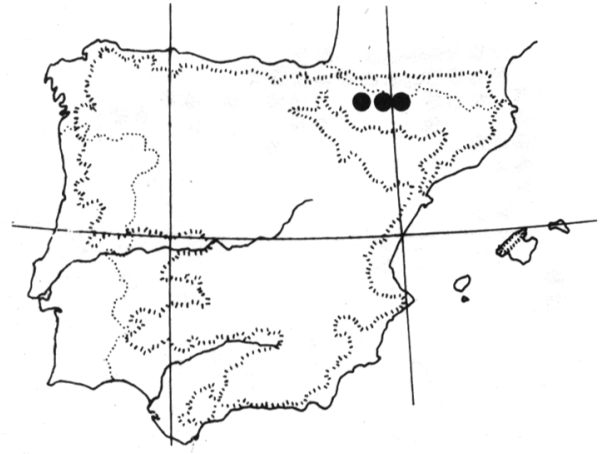

97. Cystopteris montana (Lam.) Desv.

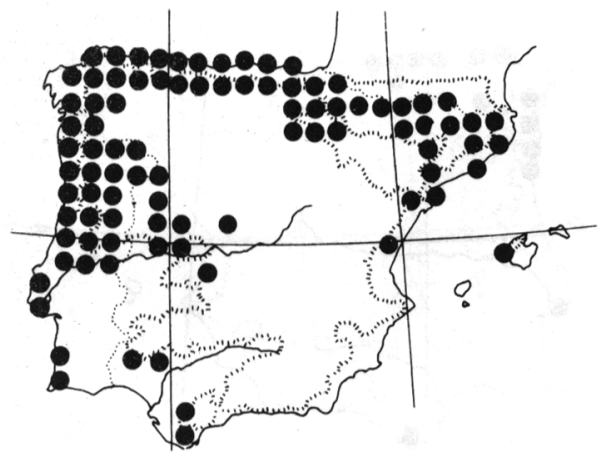

99. Polystichum setiferum (Forsskal) Woynar

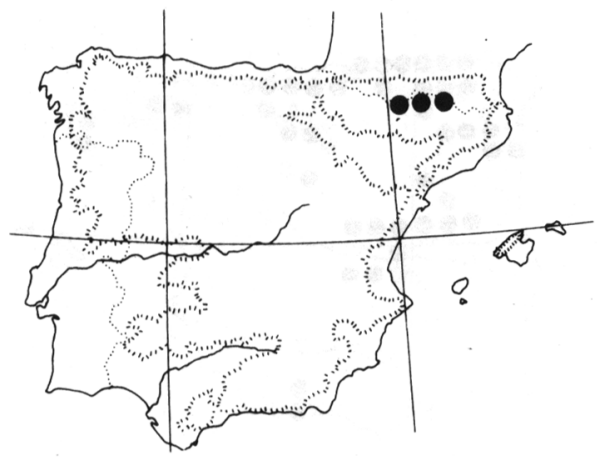

101. Polystichum braunii (Spenner) Fée

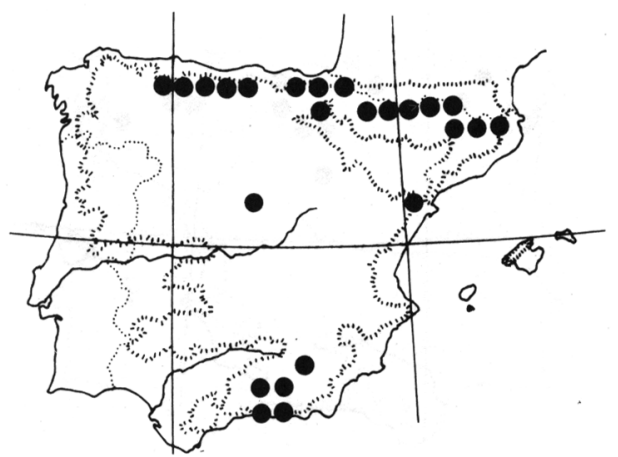

98. Polystichum lonchitis (L.) Roth

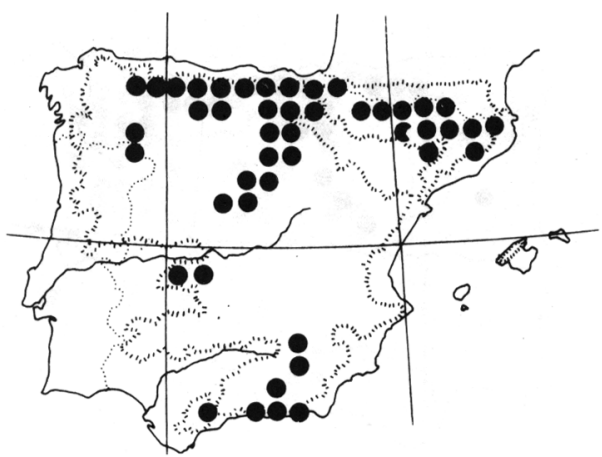

100. Polystichum aculeatum (L.) Roth

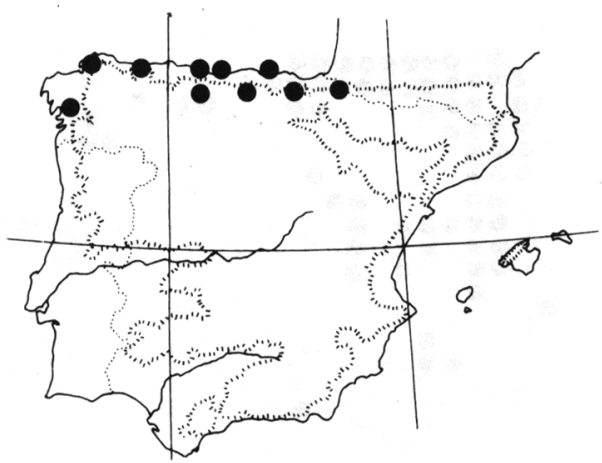

102. Dryopteris aemula (Aiton) Kuntze 


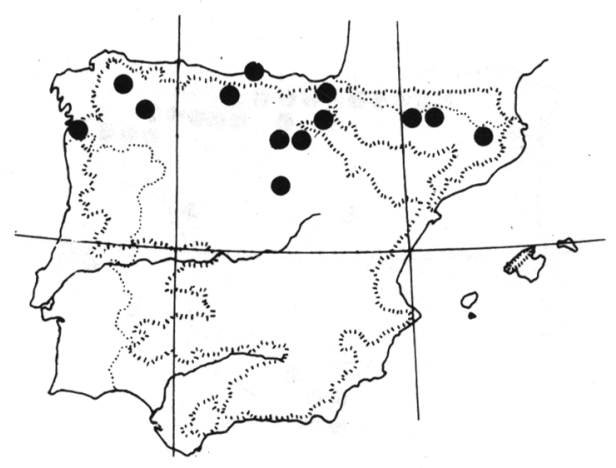

103. Dryopteris carthusiana (Vill.) H.P. Fuchs

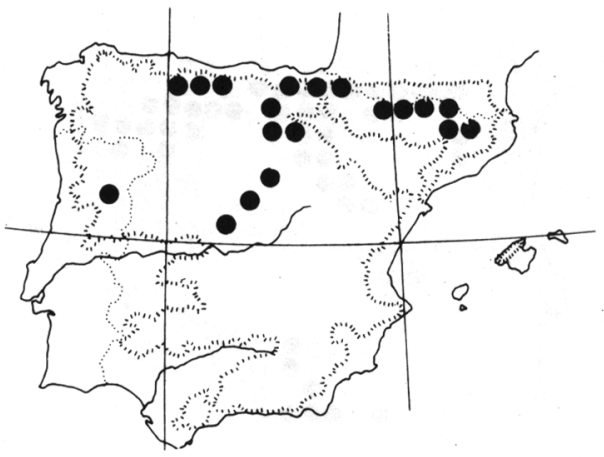

105. Dryopteris expansa (C. Presl) Fraser-Jenkins \& Jermy

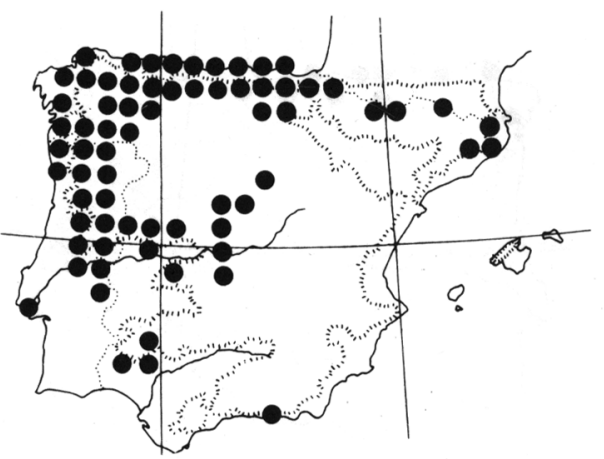

107. Dryopteris affinis (Lowe) Fraser-Jenkins subsp. affinis

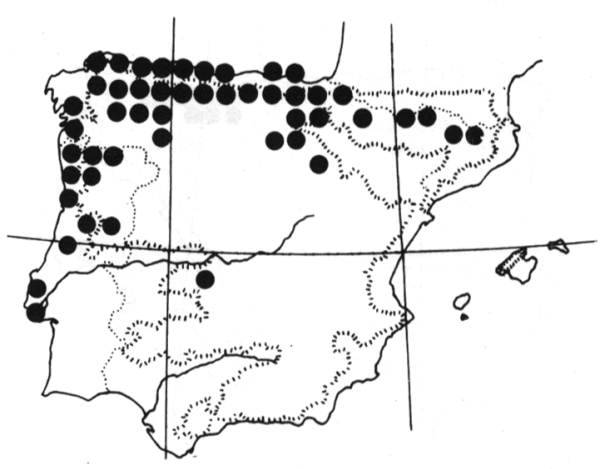

104. Dryopteris dilatata (Hoffm.) A. Gray

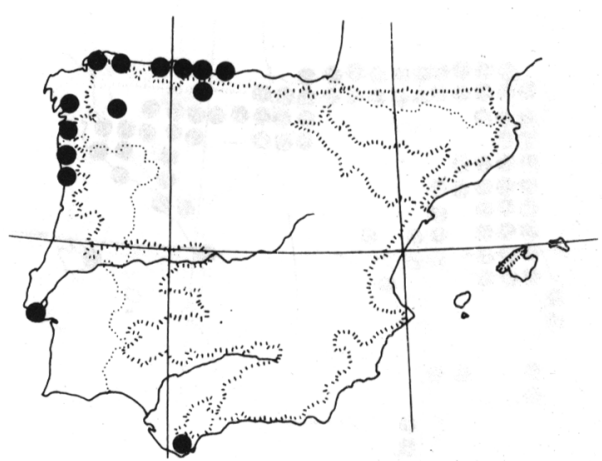

106. Dryopteris guanchica Gibby \& Jermy

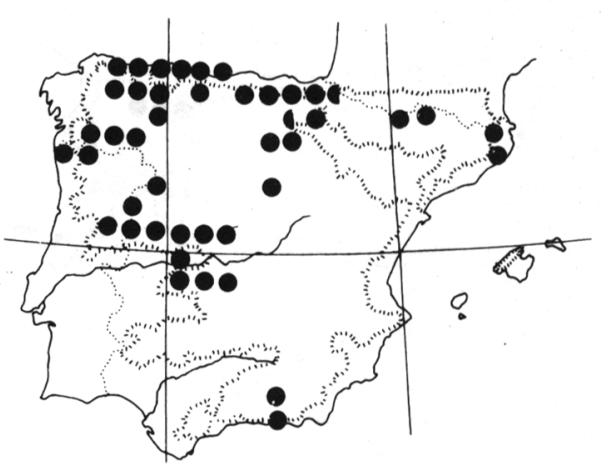

108. Dryopteris affinis (Lowe)

Fraser-Jenkins subsp. borreri (Newman) Fraser-Jenkins 


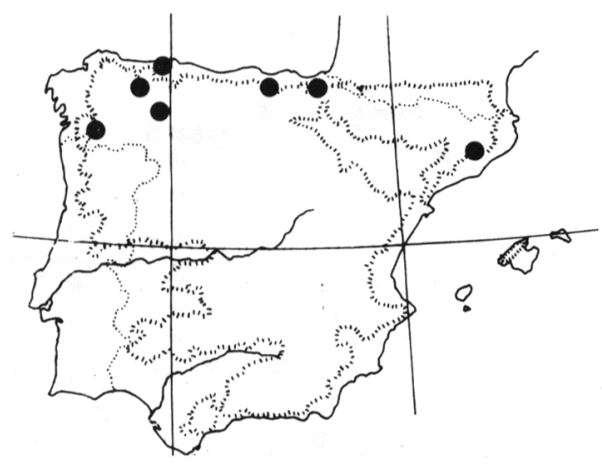

109. Dryopteris affinis (Lowe)

Fraser-Jenkins subsp. stilluppensis (Sabr.) Fraser-Jenkins

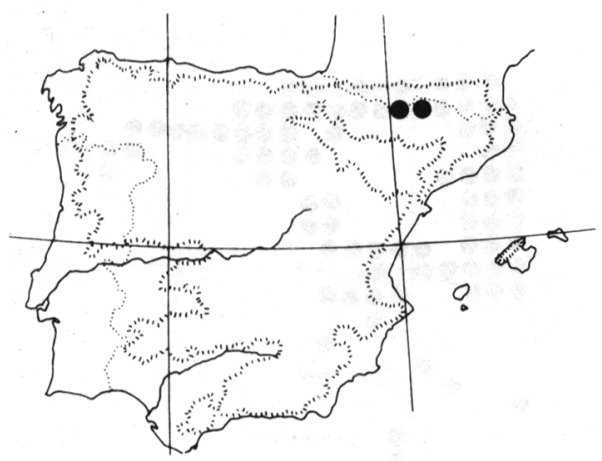

111. Dryopteris remota (Döll) Druce

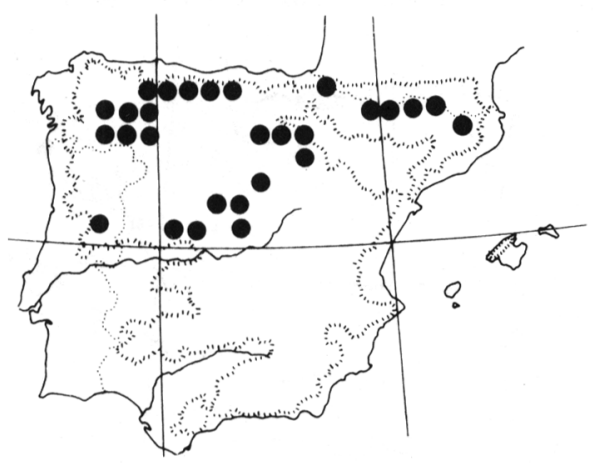

113. Dryopteris oreades Fomin

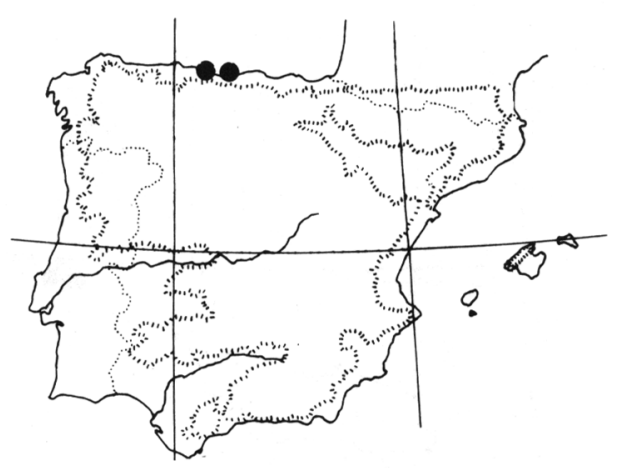

110. Dryopteris corleyi Fraser-Jenkins

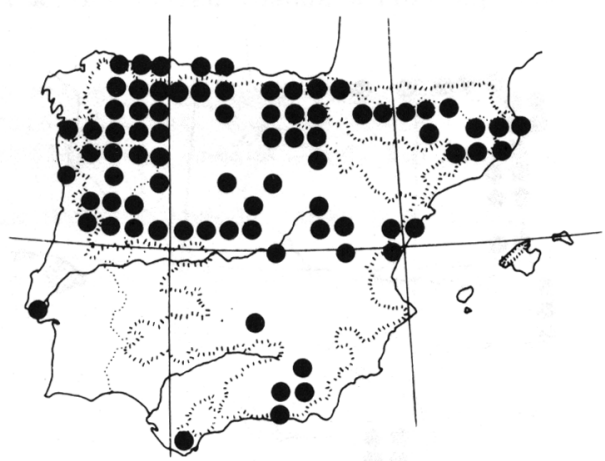

112. Dryopteris filix-mas (L.) Schott

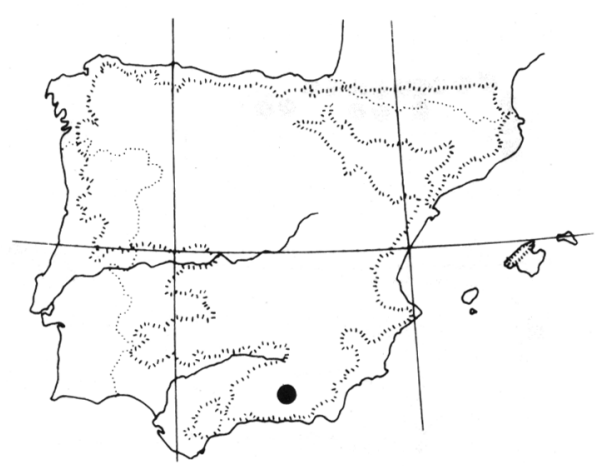

114. Dryopteris tyrrhena

Fraser-Jenkins \& Reichstein 


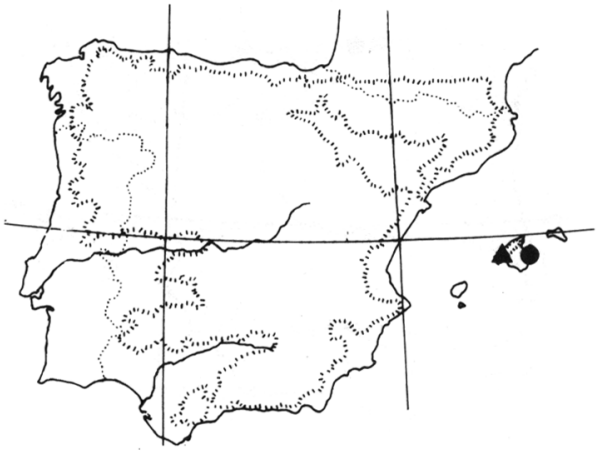

115. Dryopteris pallida (Bory) Fomin subsp. balearica (Lit.) Fr.-J. (0); subsp. pallida \& subsp. balearica (

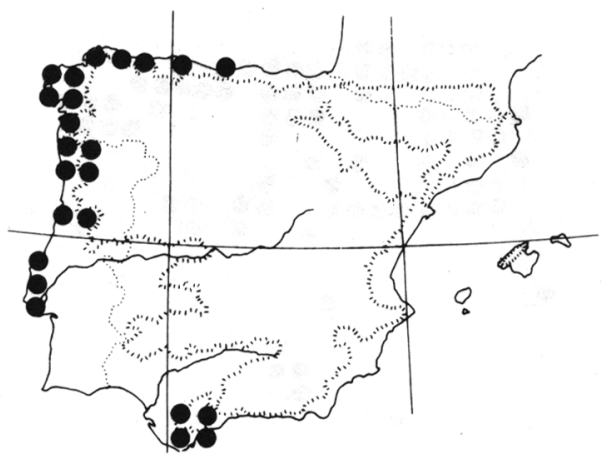

117. Davallia canariensis (L.) Sm.

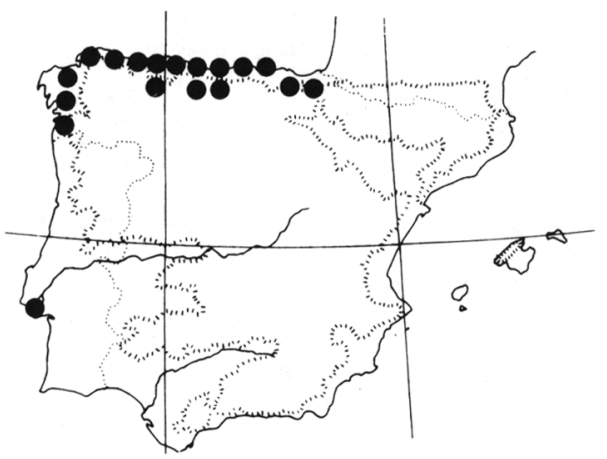

119. Woodwardia radicans (L.) Sm.

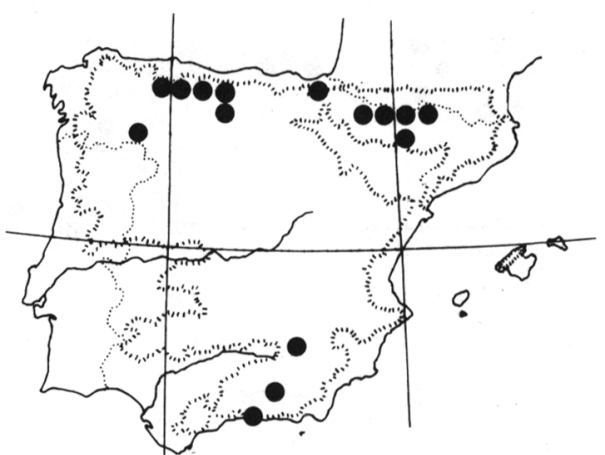

116. Dryopteris submontana

(Fraser-Jenkins \& Jermy) Fraser-Jenkins

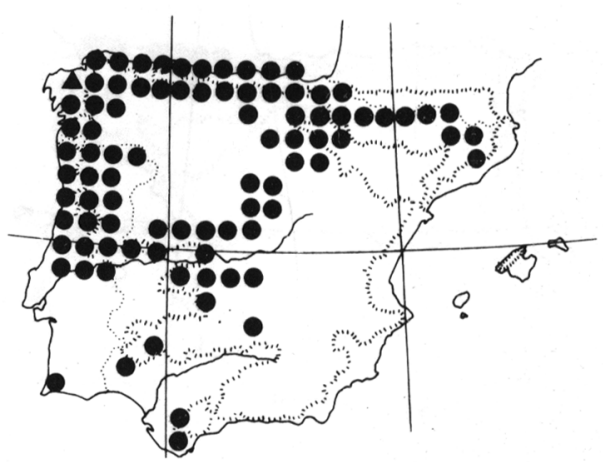

118. Blechnum spicant (L.) Roth var. spicant (1); var. homophyllum (Merino) Löve \& Löve ( $\boldsymbol{A})$.

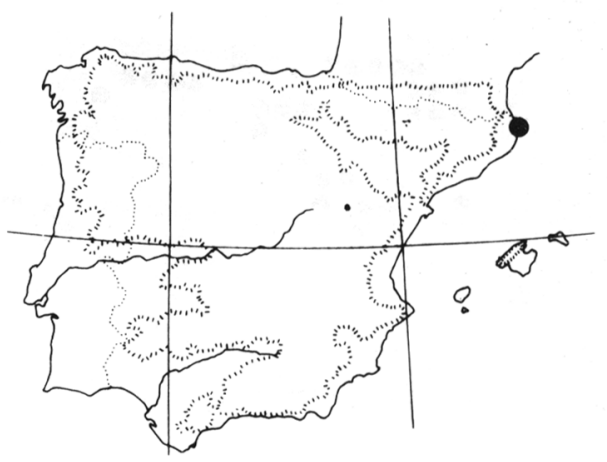

120. Salvinia natans (L.) All. 


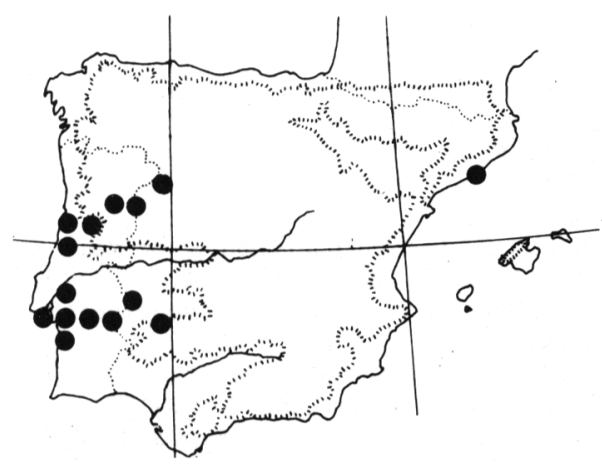

121. Azolla caroliniana Willd.

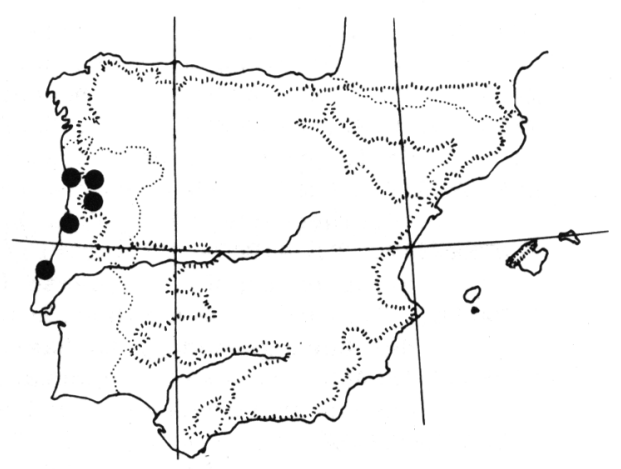

122. Azolla filiculoides Larn.

\section{BIBL IOGRAFIA}

ALCARAZ, F. \& LLIMONA, X. -1982- Estado actual del conocimiento de la pteridoflora de la provincia de Murcia. Collect. Bot. 13: 5-18.

AMICH, F. \& SANCHEZ, J. -1981- Notas corológicas. Anales Jard. Bot. Madrid $38: 306$.

BASCONES, J.C. -1982- Pteridófitos de la Navarra húmeda. Acta Bot. Malacitana $7: 199-201$.

BASCONES, J.C., EDERRA, A., PEREZ, A. \& MEDRANO, L.M. -1982- Pteridófitos de Navarra. Collect. Bot. 13:19-35.

BARRERA, I. -1980- Revisión iconográfica dos pteridófitos de Galicia. Cuadernos da área de Ciencias Bioloxicas (1).

BARRERA, I. -1980- Asplenium septentrionale Hoffm. y Asplenium cuneifolium Viv. en Galicia. Anales Real Acad. Farm. 46:125-126.

BOU, J. \& MANOBENS, R.M. -1982- Botrychium matricariifolium A. Braun ex Koch als Pirineus. Coll. Bot. 13:37-41.

CASASECAS, B., FDEZ.-DIEZ, F.J., AMICH, F., RICO, E. \& SANCHEZ, J. -1982Catálogo de las plantas vasculares de la provincia de Salamanca. I. Pteridophyta. Trab. Dep. Bot. Salamanca 10:5-27.

DIAZ, T.E. \& SALVO, A.E. -1981- Aplicación de la taxonomía numérica en pteridología: Estudio de la variabilidad en Asplenium marinum L. Trab. y monograf. 2:27-58.

FRASER-JENKINS, C. -1982- Dryopteris in Spain, Portugal \& Macaronesia. Bol. Soc. Brot. 55: 175-336.

GREUTER, W., BURDET, H. \& LONG, G. -1981- Med-Checklist I. Pteridophyta . Génève.

HORJALES, M. -1982- Notas corológicas sobre pteridófitos gallegos. Anales Jard. Bot. Madrid 38:525-526.

JALAS, J. \& SUOMINEN, J. -1972- Atlas Florae Europeae. I. Pteridophyta. Helsinki.

LAINZ, M. -1982- Mis contribuciones al estudio de la flora asturiana. Pub. Inst. Est. Ast. Oviedo.

LOIDI, A.J. -1983- Estudio de la flora y vegetación de las cuencas de los ríos Deva y Urola en la provincia de Guipuzcoa. Madrid.

LOPEZ, G. -1982- Distribución en España de Ophioglossum azoricum C. Presl. Anales Jard. Bot. Madrid 38:524-525.

LOPEZ, G. \& CASTROVIEJo, S. -1979- Dos citas nuevas de Cheilanthes. Anales Jard. Bot. Madrid 36:408. 
LORIENTE, E. -1981- Datos sobre la vegetación en Cantabria. IV. Pub. Col. Ofic. Farm. Santander.

MALATO, J. -1982- A Serra de Monchique. Flora e Vegetação. Lisboa.

MEDRANO, L.M., BASCONES, J.C., EDERRA, A. \& PEREZ, A. -1982- Pteridófitos de la provincia de Rioja. Collect. Bot. 13:43-53.

MONTSERRAT, J.M. -1982- Aportación a la flora de la Sierra de Guara (Pirineo Aragonés). Fol. Bot. Misc. 3:3-9.

MONTSERRAT, P. -1982- Comentarios sobre las investigaciones pteridológicas en España. 2a parte (1981). Collect. Bot. 13:67-84.

NAVARRO, C. -1981- Contribución al estudio de la flora y vegetación del Duranguesado y La Basturia (Vizcaya). Madrid.

PEREZ-CHISCANO, J. -1982- Aportación al estudio de los helechos de la cuenca extremeña del Guadiana. Acta Bot. Malacitana 7:193-197.

RIVAS MARTINEZ, S., COSTA, M., IZCO, J. \& SAENZ, C. -1981- Flora Matritensis, I. (Pteridophyta). Lazaroa 3:25-61.

ROMO, A. M. -1982- Pteridófitos burgaleses: Notas corológicas. Collect. Bot. 13:97-io0.

ROCHA-AFONSO, M.L. -1982- Contribuçao para o estudo do género Cystopteris en Portugal Continental e insular. Bol. Soc. Brot. 55:337-352.

SALVO, A.E. -1982- Flora pteridofítica de Andalucía. Málaga.

SALVO, A.E., ASENSI, A. \& RIVAS-MARTINEZ, S. -1981- Bibliografía pteridológica de España, Portugal e islas adyacentes. Trab. y Monograf. 2:59-104.

SANCHEZ, J. -1979- Notas florísticas para la provincia de Salamanca. Anales Jard. Bot. Madrid 36: 266.

URIBE, P.M. -1982- Pteridófitos alaveses. Collect. Bot. 13:101-117.

VILLAR, L. -1982- Dos licopodios nuevos para Andorra: Lycopodium annotinum y Lycopodium alpinum. Collect. Bot. 13:119-127. 